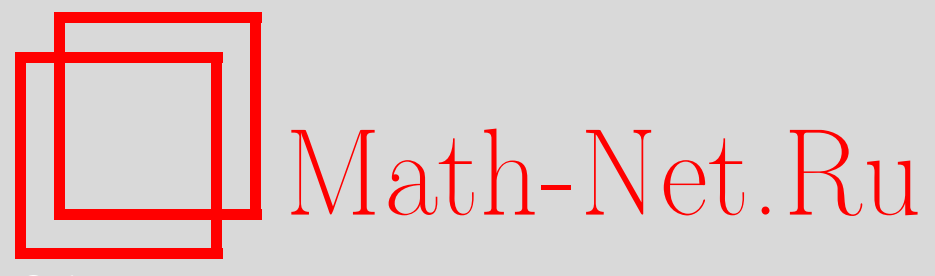

V. A. Berezin, Before getting around to do black hole physics..., Вестн. Сам. гос. техн. ун-та. Сер. Физ.-мат. науки, 2013, выпуск 4(), 138-172

DOI: https://doi.org/10.14498/vsgtu1249

Использование Общероссийского математического портала MathNet.Ru подразумевает, что вы прочитали и согласны с пользовательским соглашением

http://www . mathnet.ru/rus/agreement

Параметры загрузки:

IP : 3.85 .183 .62

26 апреля 2023 г., 13:46:20

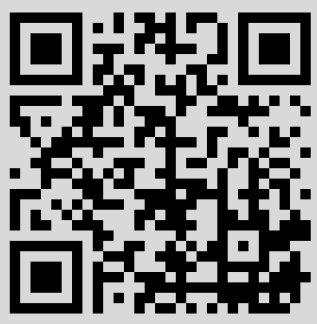




\title{
Theoretical Physics
}

\author{
UDC 517.958:524.882; MSC: 3C57; 83C10, 83C55, 83C05
}

\section{BEFORE GETTING AROUND TO DO BLACK HOLE PHYSICS...}

\section{A. Berezin}

Institute for Nuclear Research, Russian Academy of Sciences, 7a, 60th October Anniversary pr., Moscow, 117312, Russia.

E-mail: berezin@ms2.inr.ac.ru

The short history is presented of the very notion "black hole". The global geometry of the general spherically symmetric space-time is described. Einstein equations for spherical gravity are derived. The causal structure of the Schwarzschild black hole is investigated, and it is shown in details how to construct conformal Carter-Penrose diagrams that reveal visually such a structure. The Israel equations for self-gravitating thin shells are obtained and the modified gravitational Newton's law is investigated. Very simple and instructive derivation of the Vaidya metrics describing the spherically symmetric gravitating radiation is given. As an application of the theory described above the problem if the real (not virtual) static Schwarzschild observer is solved.

Keywords: General Relativity, spherical gravity, thin shells, Schwarzschild manifold, Vaidya metrics.

The essay is prepared following a series of lectures, read by the author at the Third International Conference "Mathematical Physics and Its Applications" (August 27 September 1, 2012, Samara, Russia)

Translated from Vestn. Samar. Gos. Tekhn. Univ. Ser. Fiz.-Mat. Nauki, 2013, Issue 3(32), Pages 147-184; DOI: 10.14498/vsgtu1230.

\section{Introduction}

The first mention of black holes, found till now, refers to 1784 (note: the famous "Principia ..." by Isaac Newton were published in 1687). J. Michell, the priest, reflecting on eternal, i.e., on the nature of celestial phenomena, understood that if the escape velocity on the surface of a star equals or greater than the speed of light, then such a star would be invisible for a distant observer. He called these objects dark stars and published this scientific result as a note [1]. His communication does not cause special interest-yes, there can exist the invisible stars - it is curious, and that's all! Such an ignorance is quite understandable because at the time the corpuscular theory of light prevailed, and the speed of light had no fundamental significance for physics.

The next step was done by P.-S. Laplace [2] when 1799 he published a simple (as seen nowadays) derivation of the dark stars sizes based, of course, on the Newton's gravity. By definition, the kinetic energy of the body starting to move at the escape velocity is just enough to overcome the gravitational attraction and reach an infinity. The newtonian gravitational potential outside a spherical

Victor A. Berezin (Dr. Phys. \& Math. Sci.), Senior Researcher, Theoretical Department. 
gravitating source equals

$$
U_{\text {grav }}(r)=-\frac{G M}{r},
$$

where $r$ is the distance from the gravitating center, $M$ is its mass, and $G$ is the gravitational (Newton's) constant. The additive constant of integration is chosen zero at infinity, and the "minus" sign means attraction. For the escape velocity we have

$$
\frac{m V^{2}}{2}=G \frac{M m}{R_{0}}
$$

where $R_{0}$ is the star radius. As it should be, this velocity does not depend on the mass of the "light corpuscle". Putting $v=c$ ( $c$-speed of light) we get

$$
c^{2}=\frac{2 G M}{R_{0}},
$$

and

$$
R_{0}=\frac{2 G M}{c^{2}}=R_{g} .
$$

Introduced here $R_{g}$ is called the gravitational radius. And again, this result considered as pure technical and having no fundamental value-people were not properly impressed. But we are, because it is exactly the same as that of the radius of the relativistic black hole! It is well known that by combination of the fundamental constants, speed of light $c$, gravitational $G$ and Planck constants $\hbar$, one can obtain the value of any dimensionality, so, we have to wonder at the equal coefficients only, but here it is a mere coincidence. For us, the derivation proposed by Laplace is not completely satisfactory. First of all, at the escape velocity the total (kinetic + potential) energy of the light corpuscles is exactly zero. In the case when it is negative (i.e., the star radius is less than $R_{0}$ ), then though the star is not visible for the observers at infinity it is still visible to one who sits more or less nearby. The genuine invisible objects in Newton's gravitational theory are the point sources only. Second, at the times of corpuscular theory of light nobody feels uneasy about the varying light speed-up to zero at the turning point. But very soon the concept of the wave nature of the light was adopted, and the light began considered as the ether oscillations, something very different from the rest of matter, and not obeyed the gravitational law.

The new period, though unnoticed, in the black hole theory begun in 1905 with appearing of Special Relativity [3]. The main experimentally proved postulate of this theory is that the speed of light is constant, and its value does not depend on the choice of the inertial frame. It also follows from the theory that this is the limiting speed which can not be reached by any massive body. In the last year of the 19th century M. Planck published a paper where it was proved actually, that the light is emitted and absorbed only by portions-quanta, and it was proposed the famous relation for their energy, $E=\hbar \omega$ ( $\hbar$-Planck's constant, $\omega$-angular velocity). But only after the works by A. Einstein [4,5] it become clear that the light is the set of photons particles with zero rest mass. One more Einstein's wonderful idea dated by 1905, is the mass-energy equivalence [6], which was expressed later in the revolutionary relation $E=m c^{2}$ [7]. What does it mean for us? First of all, it follows that the total energy of any body consists not only of 
masses of constituent particles, but also their kinetic energies and potential energy of mutual interactions, including exchanges by virtual (as we now know) photons that provide the electromagnetic interactions between molecules and atoms (the most impressive example is the helium nuclei). Therefore, the light as the photon flow should be subject to the universal law of gravity, and instead of mass $m$ one should use its equivalent, $E / c^{2}$.

Let us suppose that we adopted Special Relativity, i.e., the relativistic Minkowski space-time, but leaved the Newtonian gravity unchanged. Than, for the dark stars we would obtain the maximal radius $R=R_{g}$, only two times less the needed (relativistic) value, what is unimportant here. What essential is that since in this case there are no turning points for the light, the quanta emitted from the limiting surface can loose completely their initial energy $E_{0}=\hbar \omega_{0}$ only at infinity, $\omega(\infty)=0$. And such a conclusion does not depend on the initial value of frequency. In other words, if the gravitating body is compressed to the sizes less than $R_{g}$, there should appear some space region around it from where light cannot escape and, thus, reach the infinity. The world surface of the boundary of such a region is called the event horizon. It appears that the gravitating bodies change the causal structure of the space-time around them, which is considered as "a priori" given in Special Relativity. Consequently, the combining of Special Relativity and Newtonian gravity is intrinsically controversial. The positive result of our consideration is that now we have to deal not with the very surfaces of the gravitating bodies, but with the space-time regions-causal "holes", that can be properly called "black holes".

The contemporary history of "dark stars" began in 1916 with the paper by Karl Schwarzschild. It is impossible to understand the revolutionary importance of this discovery (of which he himself was unaware) without short description of the dramatic history of how General Relativity was created. Albert Einstein started to work on its formulation soon after he proposed Special Relativity, which combined the absolute space and absolute time of the Newtonian mechanics into the observer dependent four-dimensional continuum and established the special role playing by the speed of light in determining the causal relations between different events. First of all, he formulated the main physical principles the future relativistic theory of gravity should obey. These are: the (local) equivalence of gravitation and acceleration that can be traced back to proportionality of gravitational and inertial masses discovered by Galileo; determination of sources of gravity as the matter energy-momentum tensor, that replaces the mass density in the nonrelativistic Poisson equation; understanding the role of the metric tensor as the relativistic generalization of the Newtonian gravitational potential - the tensorial character of the theory was dictated by demanding its covariance with respect to general four-dimensional coordinate transformations. Einstein's close friend, Marcel Grossmann, taught him he methods of differential geometry and helped with formulation of the (then become famous) equations. The great mathematician David Hilbert obtained the very same equation by making use of the least action principle that made General Relativity, started as "some heuristical point of view" (as Einstein often stated), to become a rigorous mathematical theory. All this was finally elaborated by the end of 1915 and published in 1916. Here, it is important for us that relativistic theory of gravity turned out to be the theory of the space-time. Namely, as everyone knows, the space-time in Special Relativity is 
determined by the following line element = the squared "distance" between nearby points (the summation is supposed over two equal upper and lower indices):

$$
d s^{2}=d t^{2}-d x^{2}-d y^{2}-d z^{2}=g_{\mu \nu}(x) d x^{\mu} d x^{\nu}
$$

and, implicitly, by demanding that the metric tensor $g_{\mu \nu}$ is not changed under parallel transport. In other words, Minkowski space-time is flat, its Riemann curvature tensor is zero. We already saw (with dark stars) that the Minkowski geometry and Newtonian gravity are incompatible. Therefore, one should get rid of the flat space-time. Besides, the results obtained by Einstein and Grossmann showed that it is the coefficient of the metric tensor that become the natural generalization of the non-relativistic gravitational potential. A. Einstein was the genuine physicist, so, he was using the physical "least action principle", i.e., his aim was to construct a physical theory that would have a"good" non-relativistic limit (Poisson equation) by minimal efforts (that actually appeared enormous). That's why he was walking along the corridors of the Prussian Academy of Science and, when encountered the mathematicians, catching a button on their clothes, asked them: please, give a symmetric second rank tensor that is linear in metric second derivatives. And Marcel Grossmann showed him the one-it was the so called Ricci tensor, which is obtained from Riemann curvature tensor by contraction of two indices, provided the connections defining the parallel transport are the metric ones (i.e., he parallel transport leaves the metric tensor unchanged). With such a minimal number of postulates Einstein managed to derive the famous equations of General Relativity. D. Hilbert, on the contrary, was a genuine mathematician. His aim became to derive equations of the physical theory from the pure mathematical least action principle using variational tools. For this he needed a Lagrangian. Being excited by the early attempts of Einstein and Grossmann in constructing a relativistic theory of gravity, he has chosen for the Lagrangian the so called scalar curvature-the contracted Ricci tensor, together with the additional requirement of the metrical connections. The latter choice was dictated, may be, by his wish to have the relativistic gravitational potentials, i.e., the metric tensor components, to be the only dynamical variables of the theory. Eventually, with enormous efforts, Hilbert obtained the Einstein equations. The more complex Lagrangians, for example, in the form of non-linear scalar curvature function, lead us (if the connections is metrical) to the equations of the fourth degree by the metric tensor derivatives, and, consequently, to the non-stability of solutions. Later it was found, that varying metrics and connections independent (Palatini method) one can keep the restriction on the order of the metrics derivatives, but in that case we should use the more general Weil connections instead of the metrical connections.

Einstein reported on the new gravitational field equations at the Prussian Academy meeting on 25 of November, 1915, and then published in the Communications of this Academy. And already in two months later, in that very Communications (Sitzungsberichte der Königlich Preussischen Akademie der Wissenschaften, Berlin, 1916) there appeared the paper by K. Schwarzschild [8], where the first exact solution to these equations was derived. Now this solution is worldwide known. By this time Schwarzschild was the famous scientist, professor and academician, the Director of the Observatory in Goettingen, in the town where Hilbert and Minkowski were working. After the beginning of the World War I in 1914, he joined the German Army as a volunteer, took part in the battles in the West, 
then in the East, where he was taken lethally ill and put in the hospital. After that he, disabled, returned to Germany and died soon in 1916. Being in the hospital, K. Schwarzschild wrote three scientific papers, two of them were devoted to deriving the special solutions to the Einstein Equations (they are known at present as external and internal Schwarzschild solutions), while the third one was dealing with the Bohr-Sommerfeld quantization method. It is the first solution, as it becomes quite clear later, that turned out to be the pioneer mathematical model of a black hole. In general, derivation of the exact solutions to the Einstein equations is not a trivial task because of its nonlinearity and general covariance. The nonlinearity is an unavoidable "badness" of this theory because its the main underlying principle "any kind of energy gravitates" concerns the gravitational energy as well. This latter energy does enter the energy-momentum tensor in the right hand sides of the equations, but "encoded" in their left hand sides, which is purely geometrical. Therefore, the problem appears how to separate the physical effects from the coordinate ones, as well as their correct interpretation. The Schwarzschild metrics is the solution of the spherically symmetric vacuum Einstein equation, i.e., where energy-momentum tensor is zero. Evidently, it provides a relativistic generalization of the Newton's universal gravitational law outside of the spherical source, and, as a limiting case, the gravitational field of the point mass.

\section{General Preliminaries}

In this Section we review shortly the main notions and relations of differential geometry needed in what follows.

Construction of the space-time geometry starts with defining the squared interval between nearby points $x^{\mu}$ and $x^{\mu}+d x^{\mu}$ :

$$
d s^{2}=g_{\mu \nu}(x) d x^{\mu} d x^{\nu} .
$$

It is assumed a summation on equal upper and lower indices (Einstein's rule). When $d s^{2}>0$, the interval is called time-like, when $d s^{2}<0$-it is space-like. For null intervals $d s^{2}=0$. The same names are used for vectors contracted with themselves, $A^{\mu} A_{\mu}=g_{\mu \nu} A^{\mu} A^{\nu}$. The non-degenerate symmetric second rank tensor $g_{\mu \nu}(x)$ is called the metric tensor. By suitable choice of coordinates it can always be reduced to the diagonal form $\operatorname{diag}(+1,-1,-1,-1)$ at any given point (in other words, we will use the signature $(+---)$ ). When changing the coordinate system, $x^{\prime \mu}=x^{\prime \mu}(x)$, the metric tensor transforms in the following way,

$$
g_{\mu \nu}^{\prime}\left(x^{\prime}\right)=\frac{\partial x^{\lambda}}{\partial x^{\prime \mu}} \frac{\partial x^{\sigma}}{\partial x^{\prime \nu}} g_{\lambda \sigma}(x),
$$

as it ought to be. At the same time an arbitrary contra-variant vector (bearing an upper index) $A^{\mu}$ (in particular, the displacement vector $d x^{\mu}$ ) transforms as

$$
A^{\prime \mu}\left(x^{\prime}\right)=\frac{\partial x^{\prime \mu}}{\partial x^{\lambda}} A^{\lambda}(x),
$$

and the covariant vector (with lower index) $B_{\mu}-$ as

$$
B_{\mu}^{\prime}\left(x^{\prime}\right)=\frac{\partial x^{\lambda}}{\partial x^{\prime \mu}} B_{\lambda}(x) .
$$


It is quite clear that the conventional differential of a vector is not, in general, a vector, because

$$
d A^{\prime \mu}\left(x^{\prime}\right)=\frac{\partial x^{\prime \mu}}{\partial x^{\lambda}} d A^{\lambda}(x)+\frac{\partial^{2} x^{\prime \mu}}{\partial x^{\lambda} \partial x^{\sigma}} A^{\lambda}(x) d x^{\sigma}
$$

It follows from this that the standard, for Euclidean space and Minkowski spacetime, definition of the parallel transport for vectors, namely, $d A^{\mu}=0$ is not covariant and, thus, not good anymore. Instead, the so called covariant differential is introduced,

$$
D A^{\mu}=d A^{\mu}+\Gamma_{\nu \lambda}^{\mu} A^{\nu} d x^{\lambda}
$$

where the connection $\Gamma_{\nu \lambda}^{\mu}$ is used to compensate the second derivatives that appeared in the course of differentiation and, at the same time, to restore the vectorial (in general, tensorial) character of such an action. The covariant derivative for vectors, $A_{; \nu}^{\mu}$, is defined in a natural way:

$$
D A^{\mu}=A_{; \nu}^{\mu} d x^{\nu} \rightarrow A_{; \nu}^{\mu}=A_{, \nu}^{\mu}+\Gamma_{\nu \lambda}^{\mu} A_{\nu}^{\lambda}
$$

(the usual partial derivative is denoted by comma, while the covariant derivativeby semicolon). It can be easily checked that the covariant derivative introduced above satisfies all the conditions required for the abstract differentiation as an operator acting on the abstract mathematical objects: it is linear and obeys Leibnitz rule for the products of two vectors. By definition, the covariant derivative for scalars $\varphi$ coincides with the conventional partial derivative,

$$
\varphi ; \mu=\varphi, \mu
$$

(this is quite natural since scalars have no indices), and from it follows the differentiation rule for the covariant vectors:

$$
B_{\mu ; \nu}=B_{\mu, n u}-\Gamma_{\mu \nu}^{\lambda} B_{\lambda} .
$$

The parallel transport of a vector $A^{\mu}$ along some direction $d x^{\lambda}$ is defined by putting zero its covariant differential along this direction:

$$
D A^{\mu}=A_{; \lambda}^{\mu} d x^{\lambda}=0
$$

The time-like four-velocity vector is defined by the relation

$$
u^{\mu}=\frac{d x^{\mu}}{d s}=\frac{d x^{\mu}}{d \tau}
$$

( $d s>0, \tau$ is called the proper time), it is evident that $u^{\mu} u_{\nu}=1$. This vector is tangent to the trajectory $x^{\mu}(\tau)$. If a tangent vector undergoes the parallel transport along the trajectory, such a curve is called geodesics. Thus, the geodesic equation has the form

$$
D u^{\mu}=u_{; \nu}^{\mu} \frac{d x^{\nu}}{d \tau} d \tau=u_{; \nu}^{\mu} u^{\nu} d \tau=0
$$


It should be noted here that, in differential geometry, the metrics, i.e., the metric tensor $g_{\mu \nu}$, and connections $\Gamma_{\nu \lambda}^{\mu}$, are introduced separately and independently of each other. But, among all the connections, there exists one special-it is, first symmetric in lower indices and, second, the covariant derivative, defined by this very connection, of the metric tensor is zero,

$$
g_{\mu \nu ; \lambda}(x)=0 .
$$

It is called the metric connection, and their components-Christoffel's symbols. This metric connection is uniquely determined by the metric tensor:

$$
\Gamma_{\mu \nu}^{\lambda}=\frac{1}{2} g^{\lambda \sigma}\left(g_{\sigma \mu, \nu}+g_{\sigma \nu, \mu}-g_{\mu \nu, \sigma}\right) .
$$

In this case it is possible simultaneously to make the metric tensor diagonal and Christoffel symbols equal zero at any given point. The same can be done along the given curve. Since the connections are not tensor, such a property is not intrinsic for the space-time, but just the characteristic of the specific coordinate system. Moreover, it is impossible to do in the region surrounding the point. It appears that, transporting some vector along a small closed contour, at the end the resulting vector will be, in general, different from the initial one. The difference, of course, should be proportional to the area of the surface, bound by the contour, and some fourth rank tensor:

$$
R_{\mu \nu \lambda}^{\sigma}=\frac{\partial \Gamma_{\mu \lambda}^{\sigma}}{\partial x^{\nu}}-\frac{\partial \Gamma_{\mu \nu}^{\sigma}}{\partial x^{\lambda}}+\Gamma_{\rho \nu}^{\sigma} \Gamma_{\mu \lambda}^{\rho}-\Gamma_{\rho \lambda}^{\sigma} \Gamma_{\mu \nu}^{\rho},
$$

called the Riemann curvature tensor. The space-time is called flat if such a tensor equals zero, otherwise the space-time is curved. (Note, that in the scientific literature there is yet another definition of the curvature tensor that differs from ours by sign only. Here we follow L. D. Landau and E. M. Lifshits [9].) By contracting one upper and one lower indices in the curvature tensor one obtains the Ricci tensor,

$$
R_{\mu \nu}=\frac{\partial \Gamma_{\mu \nu}^{\lambda}}{\partial x^{\lambda}}-\frac{\Gamma_{\mu \lambda}^{\lambda}}{\partial x^{\nu}}+\Gamma_{\mu \nu}^{\lambda} \Gamma_{\lambda \sigma}^{\sigma}-\Gamma_{\mu \sigma}^{\lambda} \Gamma_{\nu \lambda}^{\sigma}
$$

(again, we follow L. D. Landau and E. M. Lifshits in choosing the indices for contraction). The last of possible contractions reduces the Ricci tensor to the scalar curvature, $R=g^{\mu \nu} R_{\mu \nu}=R_{\mu}^{\mu}$. In General Relativity the metric connection is postulated. In this case Ricci tensor is automatically symmetric.

The following combination

$$
G_{\mu \nu}=R_{\mu \nu}-\frac{1}{2} g_{\mu \nu} R
$$

is called the Einstein tensor, and his famous equations have the form:

$$
R_{\mu \nu}-\frac{1}{2} g_{\mu \nu} R=\frac{8 \pi G}{c^{4}} T_{\mu \nu}
$$

where $T_{\mu \nu}$ is the Energy-momentum tensor of the matter fields. The coefficient in the right hand side is determined in such a way that outside the source in 
the weak gravity limit the non-relativistic Poisson equation would restored. The choice of the specific combination of the geometric tensors was dictated by Bianchi identities, which after contraction take the form

$$
G_{\mu ; \nu}^{\nu}=R_{\mu ; \nu}^{\nu}-\frac{1}{2} R, \mu=0 .
$$

From this the continuity equation for the energy-momentum tensor follows automatically:

$$
T_{\mu ; \nu}^{\nu}=0
$$

and this is analogous to the situation in electrodynamics where the continuity equation for the electric current follows directly from Maxwell equations. The continuity equation (1) can be also obtained independently as a consequence of the general covariance, i.e., the invariance of the matter action functional under arbitrary (but sufficiently smooth) coordinate transformations.

\section{Spherical Gravity}

3.1. Invariants and Global Structure. The spherically symmetric metrics in any non-singular point can be written in the form (reminder: we are using the signature $(+---))$

$$
d s^{2}=\gamma_{i k} d x^{i} d x^{k}-R^{2}(x) d \sigma^{2}=A d t^{2}+2 H d t d q-B d q^{2}-R^{2} d \sigma^{2},
$$

where $d \sigma^{2}=d \vartheta^{2}+\sin ^{2} \vartheta d \varphi$ is the line element of the 2-dim unit sphere, $R$ is the radius of the sphere with the area $4 \pi R^{2}$, and the metric coefficients $A$, $H, B$ and the radius $R$ are functions of some temporal coordinate $t$ and some spatial coordinate $q$. For a given manifold the radius $R(t, q)$ is and invariant, and coefficients $A, H$ и $B$ of a 2-dim manifold are determined up to the arbitrary coordinate transformations

$$
\tilde{t}=\tilde{t}(t, q), \quad \tilde{q}=\tilde{q}(t, q),
$$

that leave unchanged the explicit spherically symmetry of the metrics (2). Such a freedom (gauge freedom) can be used for fixing the coordinate system in the most suitable way need to solve a specific problem. For example, one can always put the line element to the diagonal form $(H=0)$ and still have one more degree of freedom. There is another very important choice-the so-called double-null coordinates $(u, v)$, when $A=B=0$. Then the line element (2) takes the form

$$
d s^{2}=2 H(u, v) d u d v-R^{2}(u, v) d \sigma^{2}=0,
$$

and, the only left, metric coefficient is determined up to the substitution $\tilde{u}=\tilde{u}(u)$, $\tilde{v}=\tilde{v}(v)$. We therefore see that the metrics of the spherically symmetric spacetime is locally defined actually (up to gauge transformations) by only two functions of two variables. It is natural to choose the invariant radius $R$, as one of them. It is quite desirable that the second function appeared to be also invariant. From the pure geometrical reasons one can suppose that for such an invariant there can serve the (Lorentzian) square of the normal vector to the surfaces of constant radii, $R=$ const, namely, the vector $R,_{i}(i=0,1)$ :

$$
d R=\left(\partial_{i} R d x^{i}\right)=R,{ }_{i} d x^{i}=0 .
$$


Its square equals

$$
\Delta=R,{ }_{i} R,{ }_{k} \gamma^{i k}
$$

where $\gamma^{i k}$ is inverse to the two-dimensional metric tensor $\gamma_{i k}$. When using doublenull coordinates, then

$$
\Delta=\frac{2}{H} R,{ }_{u} R, v
$$

and it is clear now that the knowledge of two invariant functions $R$ and $\Delta$ really determines the metrics (2) up to gauge transformations.

The function $\Delta(t, q)$ brings a nontrivial information about a space-time structure. Indeed, in the flat Minkowski space-time $\Delta \equiv 1$, all the surfaces $R=$ const are time-like and therefore, $R$ can be chosen as spatial coordinate $q=R$ on the whole manifold. But in the curved space-time $\Delta$ is no more constant and can in general be both positive and negative. The region with $\Delta<0$ is called the $R$ region, and the radius can be chosen as a radial coordinate $q$. In the region with $\Delta>0$ the surfaces $R=$ const are space-like (the normal vector is time-like), and the radius $R$ can be chosen as a time coordinate $t$. Such regions are called the $T$-regions. The $R$ - and $T$-regions were introduced by I. D. Novikov [10]. But this is not the whole story. It is easy to show that we can not get $\dot{R}=0$ ("dot" means a time derivative) in a $T$-region. Hence it must be either $\dot{R}>0$ (such a region of inevitable expansion is called $T_{+}$-region) or $\dot{R}<0$ (inevitable construction, a $T_{--}$ region). The same holds for $R$-regions. They are divided in two classes, those with $R^{\prime}>0$ ("prime" stands for a spatial derivative) which are called $R_{+}$-regions, and $R_{-}$-regions with $R^{\prime}<0$. These, $R$ - and $T$-regions are separated by the surfaces $\Delta=0$ which are called the apparent horizons. The apparent horizons can be null, time-like or space-like. We see, therefore, that the curved spherically symmetric space-time can have a rather complex structure and consist of a set of $\mathrm{R}_{ \pm^{-}}$and $\mathrm{T}_{ \pm}$-regions separated by apparent horizons $\Delta=0$.

3.2. Vectorial Equation It appeared that Einstein equations in the case of spherical symmetry can be rewritten in terms of the invariants introduced above, $R(t, q)$ и $\Delta(t, q)$. For this, let us write the metrics in the form

$$
d s^{2}=\gamma_{A B} d x^{A} d x^{B}-R^{2}(x)\left(d \vartheta^{2}+\sin ^{2} \vartheta d \varphi^{2}\right),
$$

where $\gamma_{A B}$ is a 2-dim metric tensor, and $A, B=0,1$. The nonzero components of a Ricci tensor equal

$$
R_{A}^{B}=\tilde{R}_{A}^{B}-2 \gamma^{B C} \frac{R_{\mid A C}}{R}, \quad R_{2}^{2}=R_{3}^{3}=-\frac{1}{R^{2}}\left(\gamma^{C D} R R_{\mid C D}+\Delta+1\right) .
$$

Here $\tilde{R}_{A}^{B}$ is a Ricci tensor for a 2-dim manifold with a metric tensor $\gamma_{A B}$ and its inverse $\gamma^{C D}$, the vertical line denotes a covariant derivative with respect to the metric connection (remember that $\gamma_{A B \mid C}=0$ ), and comma means, as usual, a partial derivative. The Einstein tensor reads now:

$$
\begin{gathered}
G_{A}^{B}=-2 \gamma^{B C} \frac{R_{\mid A C}}{R}+\delta_{A}^{B}\left(2 \gamma^{C D} \frac{R_{\mid C D}}{R}+\frac{\Delta+1}{R^{2}}\right), \\
G_{2}^{2}=G_{3}^{3}=\gamma^{C D} \frac{R_{\mid C D}}{R}-\frac{1}{2} \tilde{R}
\end{gathered}
$$


where $\tilde{R}$-2-dim scalar curvature, and we took into account that 2-dim Einstein tensor is identically zero. Constructing the following combination

$$
\delta_{A}^{B} G_{C}^{C}-G_{A}^{B}=2 \gamma^{B C} \frac{R_{\mid A C}}{R}+\frac{\Delta+1}{R^{2}} \delta_{A}^{B}
$$

and noting that

$$
\Delta, A=2 \gamma^{D C} R,{ }_{D} R_{\mid A C},
$$

we get, finally, the vectorial equation

$$
(R(\Delta+1)),_{A}=8 \pi G R^{2}\left(T_{C}^{C} R,{ }_{A}-T_{A}^{B} R, B\right) .
$$

The third equation for $A \neq B$ is, essentially, scalar:

$$
\gamma^{A C} R_{\mid C B}=-4 \pi G R T_{B}^{A}
$$

It can also be obtained as the integrability condition for the vectorial equation with making use of the Bianchi identities (or the continuity equation) and the remaining $\left(\begin{array}{l}2 \\ 2\end{array}\right)$ (scalar) of Einstein equations. The continuity equation takes now the form

$$
T_{A \mid B}^{B}+2 \frac{R, B}{R} T_{A}^{B}=2 \frac{R, A}{R} T_{2}^{2} .
$$

These equations were first derived in [11], then generalized to the spheres of arbitrary dimension [12] and to the arbitrary homogeneous and isotropic spaces [13].

Carter-Penrose Conformal Diagrams. The causal structure of a geodesically complete spherically symmetric space-time is seen best using Carter-Penrose conformal diagrams, where each point represents a sphere, and infinities are brought to finite distances. Since any two-dimensional space-time is conformally flat (at least locally), the full diagram is, actually, a set of those for 2-dim Minkowski space-time. In order to understand, how the latter can be constructed, let us first transform the conventional 2-dim Minkowski metrics

$$
d s^{2}=d t^{2}-d x^{2}
$$

to the double-null coordinates $u=t-x$ (retarded time) and $v=t+x$ (advanced time), then

$$
d s^{2}=d u d v
$$

Let us take as a rule that on our diagrams the temporal coordinate increases from down to up, the spatial coordinate-from left to right, and the null curves ( $u=$ const, $v=$ const) are represented by the straight lines with the slope $\pm 45^{\circ}$. After one more transformation,

$$
u^{\prime}=\arctan u, \quad v^{\prime}=\arctan v, \quad-\pi / 2 \leqslant u^{\prime} \leqslant \pi / 2, \quad-\pi / 2 \leqslant v^{\prime} \leqslant \pi / 2
$$

we get

$$
d s^{2}=\Omega^{2} d s^{\prime 2}, \quad \Omega=\cos ^{-1} u^{\prime} \cos ^{-1} v^{\prime}, \quad d s^{\prime 2}=d u^{\prime} d v^{\prime}=d t^{\prime 2}-d x^{\prime 2} .
$$


Formally, the metrics $d s^{2}$ is the same as the initial one, but now the coordinates $\left(u^{\prime}, v^{\prime}\right)$ and $\left(t^{\prime}, x^{\prime}\right)$ run finite intervals. The Carter-Penrose diagram for the complete 2-dim Minkowski space-time, $(-\infty<t<\infty,-\infty<x<\infty)$ is shown in Fig. 1. Here $J^{ \pm}\left(J^{\prime \pm}\right)$ are future, $\left(v^{\prime}\left(u^{\prime}\right)=\pi / 2, v(u)=\infty\right)$, and past, $\left(u^{\prime}\left(v^{\prime}\right)=-\pi / 2\right.$, $u(v)=-\infty)$, null infinities, $i_{ \pm}$are future and past temporal infinities, $\left(t^{\prime}= \pm \pi / 2\right)$, and $i_{0}\left(i_{0}^{\prime}\right)$-spatial infinities, $\left(x^{\prime}= \pm \pi / 2, x= \pm \infty\right)$. For the four-dimensional Minkowski space-time this diagram should be cut along the time-like line $r=0$, which becomes now a part of a boundary instead of that at $(-\infty)$, see Fig. 2 . The time-like surfaces of constant radii are shown by dashed curves. In general, such an orientation of the triangle corresponds to those part of the full diagram that represent the $R$-regions. For $T$-regions, the figure should turn on the straight angle $90^{\circ}$ together with the dashed curves which become the space-like ones. The apparent horizons (null, time-like or space-like ones) as well as infinities and singularities serve as boundaries for different regions of the manifold. The spacetimes are called geodesically complete if all the null and time-like geodesics start and end either at infinities or at the singularities.

\section{Schwarzschild Black Hole}

4.1. Solution. Now we have everything at hand for investigating the Schwarzschild geometry. Let us remind that, by definition, this is the space-time outside a "point-like" gravitating source (it will become clear very soon why quotation marks are used here).

The starting point is calculation of the invariant $\Delta$. Since the energy-momentum tensor is now zero, one gets immediately from the vectorial equation (3)

$$
\Delta=-1+A / R
$$

where $R(t, q)$ is the radius, and $A$-integration constant. Karl Schwarzschild has chosen the radius as a spatial (radial) coordinate, and the time coordinate orthogonal to it. We will do now the same remembering however, that such a choice is possible only in $R$-regions. In this case it follows from definitions that

$$
\Delta=g^{00} \dot{R}^{2}+g^{11} R^{\prime 2}=g^{11},
$$

and one finds

$$
g_{11}=1 / g^{11}=1 / \Delta .
$$

We will not calculate here the remaining metric coefficient $g_{00}=1 / g^{00}$, but say that, with the supposed form of the metrics the Einstein equations contain its spatial derivatives (of first and second order) only, so $g_{00}$ itself is determined up to the arbitrary factor depending on the time coordinate, which can be "absorbed" by redefinition of the latter. This means that in the $R$-region the metrics is automatically static (Schwarzschild just supposed such a property). Besides, it appears, that

$$
g_{00}=-\Delta=1-A / r
$$

(in what follows we will use a small letter $r$ for the radius as a coordinate). Thus, we reproduce the famous metrics

$$
d s^{2}=(1-A / r) d t^{2}-\frac{d r^{2}}{1-A / r}-r^{2}\left(d \vartheta^{2}+\sin ^{2} \vartheta d \varphi^{2}\right) .
$$




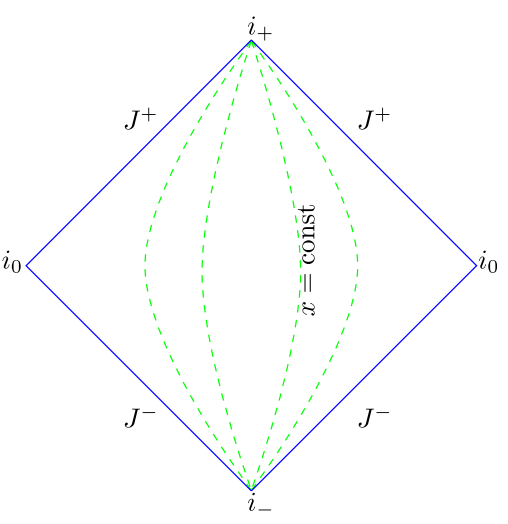

Figure 1. Carter-Penrose diagram for the complete two-dimensional Minkowski space-time $(-\infty<t<\infty,-\infty<x<\infty)$

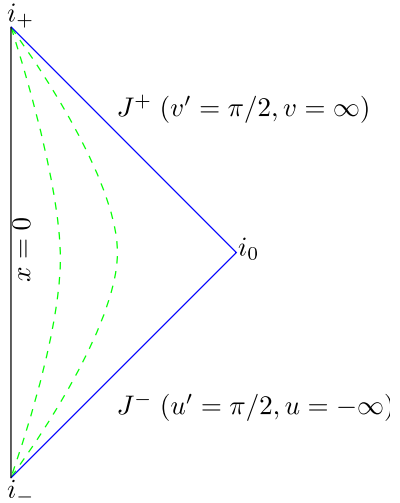

Figure 2. Carter-Penrose diagram for 4dim Minkowski space-time

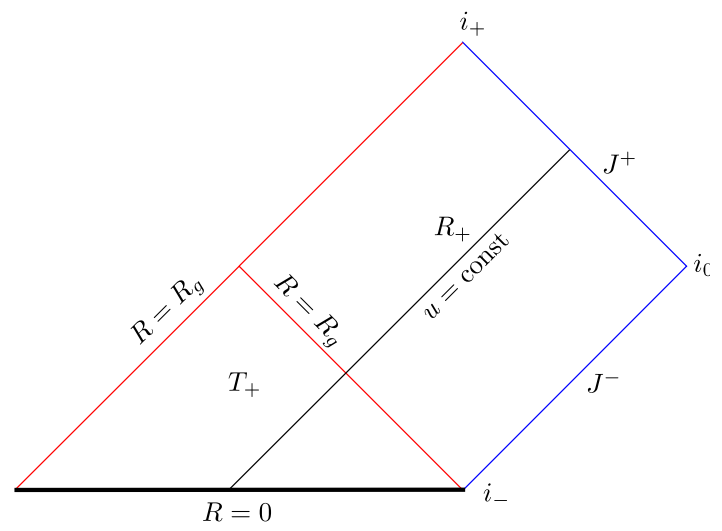

Figure 3. Carter-Penrose diagram covered by retarded Finkelstein coordinates

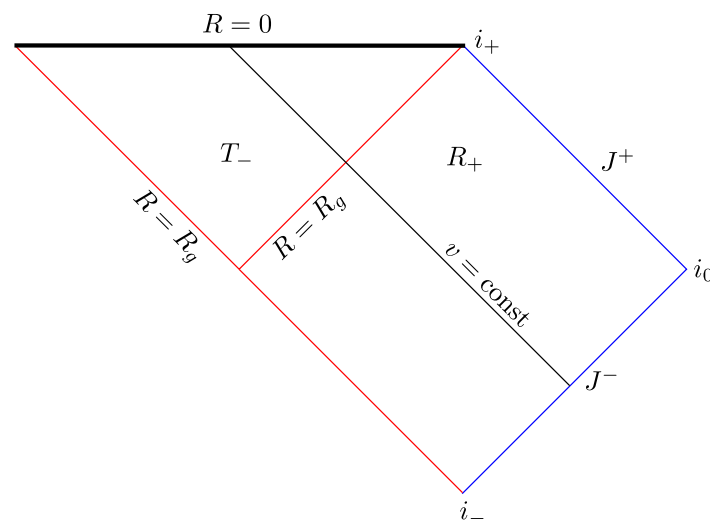

Figure 4. Carter-Penrose diagram covered by advanced Finkelstein coordinates 


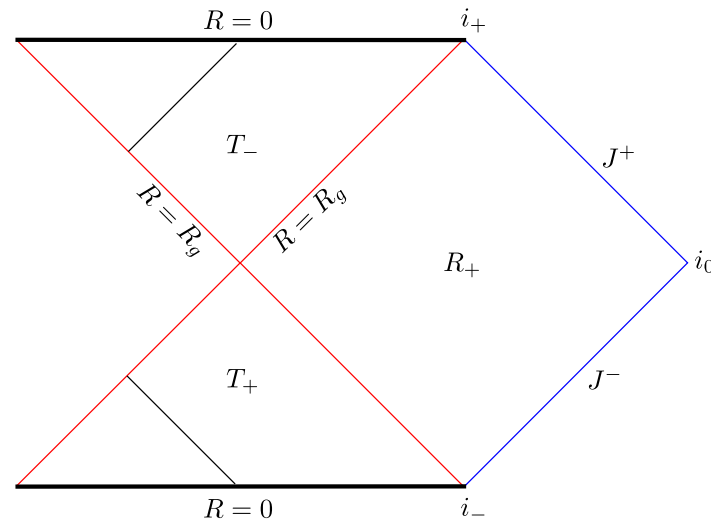

Figure 5. Combination of two diagrams (see Fig. 3 and Fig. 4)

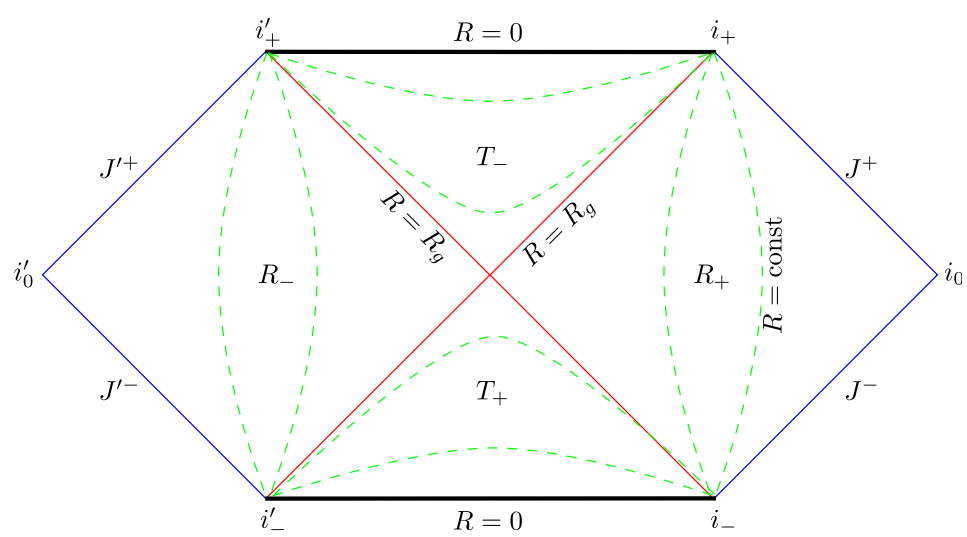

Figure 6. Carter-Penrose diagram for the geodesically complete Schwarzschild space-time

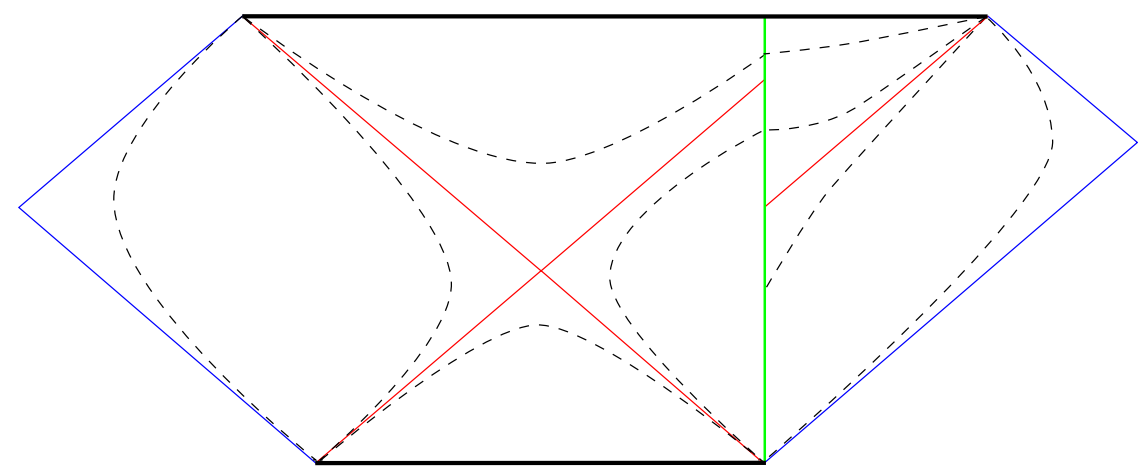

Figure 7. Carter-Penrose diagram for infinite motion when $\Delta m>0 \Longrightarrow \sigma_{\text {in }}(\infty)=\sigma_{\text {out }}(\infty)=+1$ 


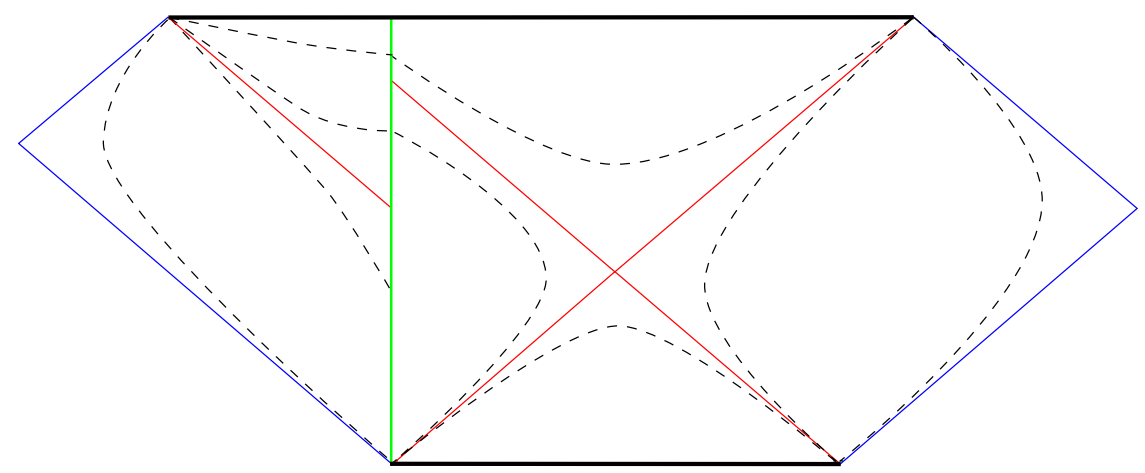

Figure 8. Carter-Penrose diagram for infinite motion when $\Delta m<0 \Longrightarrow \sigma_{\text {in }}(\infty)=\sigma_{\text {out }}(\infty)=-1$

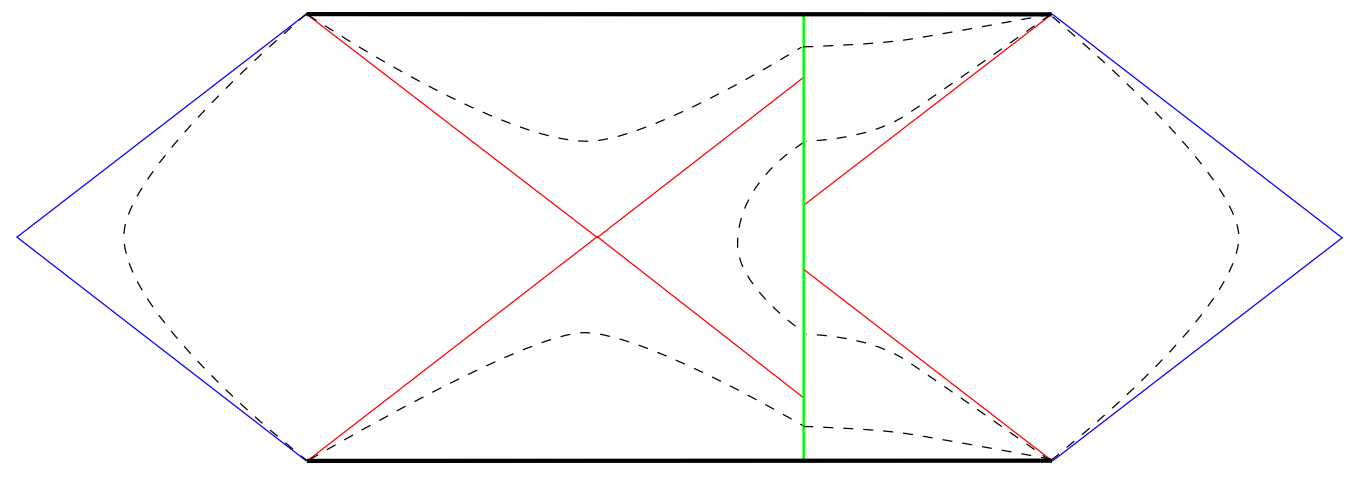

Figure 9. Carter-Penrose diagram for the finite motion of the shell with the turning point at $\rho=\rho_{0}$ and $\Delta m>0 \Longrightarrow \sigma_{\text {in }}\left(\rho_{0}\right)=+1, \sigma_{\text {out }}\left(\rho_{0}\right)=+1$

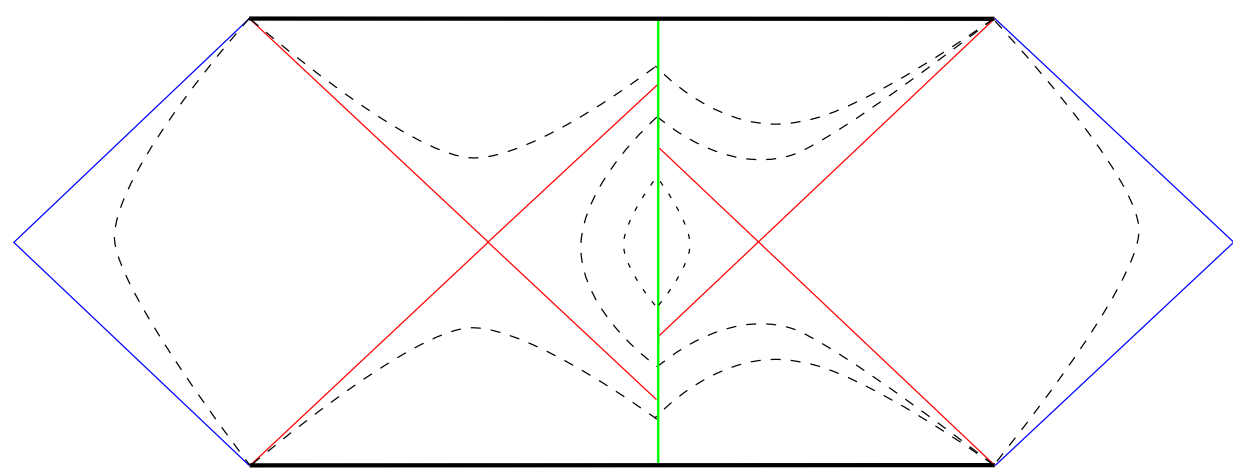

Figure 10. Carter-Penrose diagram for the finite motion of the shell with the turning point at $\rho=\rho_{0}$ and $\Delta m>0 \Longrightarrow \sigma_{\text {in }}\left(\rho_{0}\right)=+1, \sigma_{\text {out }}\left(\rho_{0}\right)=-1$ 


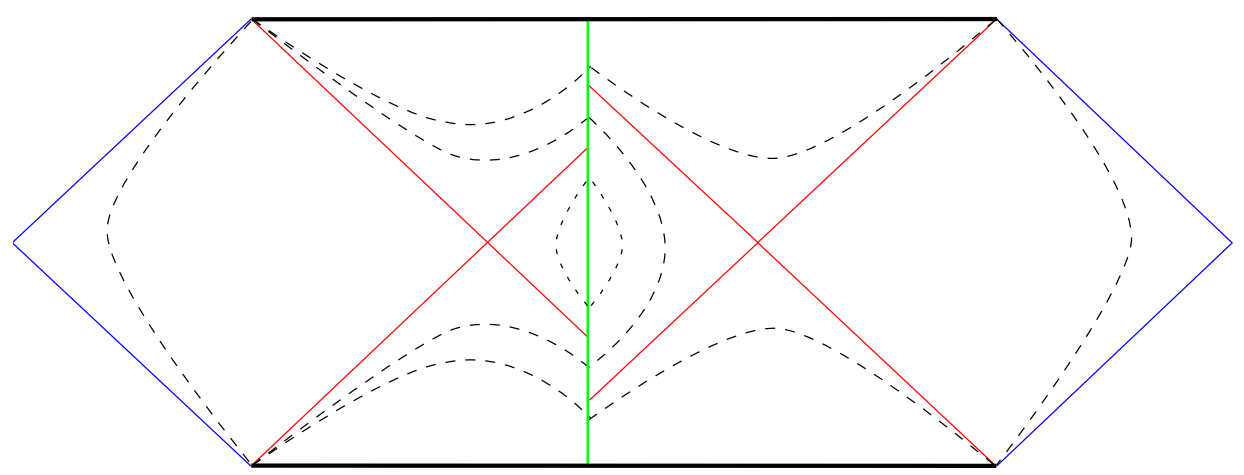

Figure 11. Carter-Penrose diagram for the finite motion of the shell with the turning point at $\rho=\rho_{0}$ and $\Delta m<0 \Longrightarrow \sigma_{\text {in }}\left(\rho_{0}\right)=+1, \sigma_{\text {out }}\left(\rho_{0}\right)=-1$

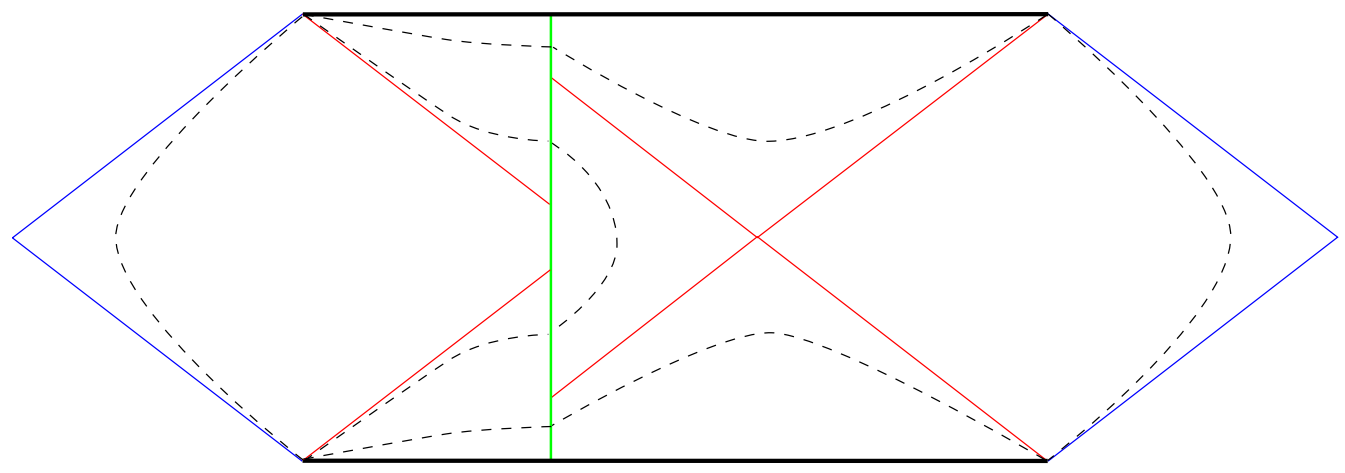

Figure 12. Carter-Penrose diagram for the finite motion of the shell with the turning point at $\rho=\rho_{0}$ and $\Delta m<0 \Longrightarrow \sigma_{\text {in }}\left(\rho_{0}\right)=-1, \sigma_{\text {out }}\left(\rho_{0}\right)=-1$

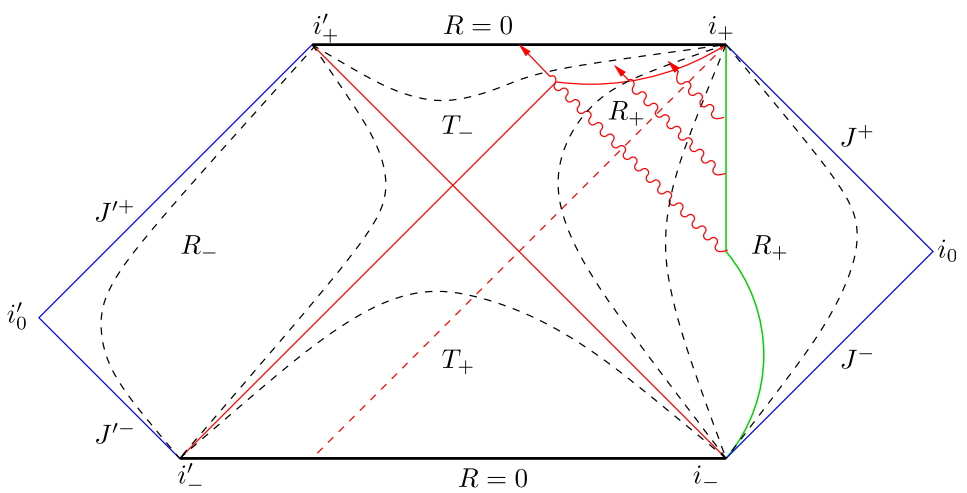

Figure 13. Carter-Penrose conformal diagram for the geodesically complete space-time under consideration, each point represents a sphere 
Before going further, let us determine the constant of integration.

4.2. Source. Let us remind that the Schwarzschild metrics depends, actually, on only one function,

$$
F=-\Delta=1-A / r
$$

where $A$ is an integration constant. In the Introduction we wrote it as

$$
A=\frac{2 G m}{c^{2}}
$$

where $G$ is the gravitational constant, $c$ is the speed of light, and $m$ some entity with dimension of mass. It is in this form that the integration constant was written by Schwarzschild, $m$ being considered as the mass of the gravitating source. How he recognized this?-from the analysis of non-relativistic transition of the Einstein equations to the Poisson equation (formally, by putting $c \rightarrow \infty$ ). Since the thorough investigation is too long and cumbersome we confine ourselves by comparison of Lagrangians for particles moving in an external gravitational field, both in the non-relativistic and (properly approximated) relativistic mechanics.

In the non-relativistic theory the Lagrangian for a particle of mass $\mu$ is the following

$$
L=T-U+\text { const }=\frac{\mu \vec{V}^{2}}{2}+\frac{G m \mu}{r}+\text { const },
$$

where $T$ is its kinetic, and $U$-potential energy. In the relativistic theory the action functional equals

$$
\begin{aligned}
& S=-\mu c \int d s=-\mu c \int \sqrt{g_{\alpha \beta} d x^{\alpha} d x^{\beta}}= \\
&=-\mu c \int \sqrt{g_{00} c^{2} d t^{2}+2 g_{0 i} c d t d x^{i}+d \vec{x}^{2}}= \\
&=-\mu c^{2} \int \sqrt{g_{00}+2 g_{0 i} \frac{V^{i}}{c}+\frac{\vec{V}^{2}}{c^{2}}} d t
\end{aligned}
$$

where $V^{i}$ is a component of the 3-dim particle velocity $\vec{V}$. The Lagrangian, thus, takes the form

$$
L=-\mu c^{2} \sqrt{g_{00}+2 g_{0 i} \frac{V^{i}}{c}+\frac{\vec{V}^{2}}{c^{2}}} .
$$

Far away from the sources, $(r \rightarrow \infty)$, the metrics becomes almost Minkowskian, i.e., $g_{00} \rightarrow 1, g_{0 i} \rightarrow 0, g_{i i} \rightarrow-1$, and in the non-relativistic limit $\left(V^{2} / c^{2} \ll 1\right)$ one gets

$$
L \approx-\mu c^{2} \sqrt{g_{00}-\frac{\vec{V}^{2}}{c^{2}}} \approx-\mu c^{2}\left(\sqrt{g_{00}}-\frac{\vec{V}^{2}}{2 c^{2}}\right) .
$$

Since $g_{00}=1-A / r$, then

$$
L \approx-\mu c^{2}\left(1-\frac{A}{2 r}-\frac{\vec{V}^{2}}{c^{2}}\right) \approx-\mu c^{2}+\frac{\mu c^{2} A}{2 r}+\frac{\mu \vec{V}^{2}}{2} .
$$


Comparing with the Eqn. (5), we see that the relativistic Lagrangian, Eqn. (6), becomes its non-relativistic counterpart, Eqn. (5), if in the latter one put an arbitrary additive constant equal $-\mu c^{2}$, and the integration constant- $A=2 G \mathrm{~m} / \mathrm{c}^{2}$, where $m$ is the mass of gravitating source. Now it is quite clear that the remote observer measures the gravitational (inertial) mass of the source.

4.3. Global Geometry: Construction of Carter-Penrose Diagrams. Let us write the Schwarzschild metrics once more $(c=1)$

$$
d s^{2}=(1-2 G m / r) d t^{2}-\frac{d r^{2}}{1-2 G m / r}-r^{2} d \sigma^{2} .
$$

It describes a curved space-time, this follows, e.g., from the fact that the distance between two points in the radial direction is greater that the difference of finite and initial values of radii:

$$
d l=\frac{d r}{\sqrt{1-2 G m / r}} ; \quad \Delta l=\int_{r_{2}}^{r_{1}} \frac{d r}{\sqrt{1-2 G m / r}}>\Delta r=r_{1}-r_{2} .
$$

Let us calculate what is the period of time (measured by distant observer) a beam of light needs for traveling:

$$
\begin{gathered}
d s^{2}=0 ; \quad d t=\frac{d r}{1-2 G m / r} \\
T_{12}=\int_{r_{2}}^{r_{1}} \frac{r d r}{r-2 G m}=r_{1}-r_{2}+2 G m \ln \frac{r_{1}-2 G m}{r_{2}-2 G m} .
\end{gathered}
$$

This expression diverges at $r_{2} \rightarrow r_{g}$. It means that even the light moving with the maximal possible speed, needs and infinite time to reach the Schwarzschild sphere. It seems that everything is nice! The collapsing star would be freezed near its gravitational radius. And all the problems are solved, we are not forced to think about what is going inside. But, ... ! It appeared that the freely falling observer reaches the Schwarzschild sphere for finite period of its proper time. Therefore, the manifold covered by the metrics, Eqn. (7), is not geodesically complete. This part of the space-time is, actually, the $R$-region, bounded by the apparent horizons, $\Delta=0$, at $R=r_{g}=2 G m$. Since in our case this invariant depends only on radius, then the apparent horizons are null. Indeed,

$$
g^{A B} \Delta,{ }_{A} \Delta,{ }_{B}=\left(\frac{d \Delta}{d R}\right)^{2} g^{A B} R,{ }_{A} R,,_{B}=\left(\frac{d \Delta}{d R}\right)^{2} \Delta=0 .
$$

In order to understand, how the "patch" of the Carter-Penrose diagram, representing this $R$-region, looks like, let us introduce, following Finkelstein [14], the so called "tortoised" cordinate $r^{\star}$ in the following way. First, put the two-dimensional part of the Schwarzschild metrics into the conformally flat form

$$
d s_{2}^{2}=\left(1-r_{g} / r\right)\left(d t^{2}-\frac{d r^{2}}{\left(1-r_{g} / r\right)^{2}}\right)=\left(1-r_{g} / r\right)\left(d t^{2}-d r^{\star 2}\right),
$$

where

$$
d r^{\star}= \pm \frac{d r}{1-r_{g} / r}, \quad r^{\star}= \pm r_{g}\left(r / r_{g}+\ln \left|r / r_{g}-1\right|\right) .
$$


Evidently, in the $R_{+}$-region one should choose the sign "+". At the apparent horizons $r^{\star}=-\infty$. Thus, in the conformally flat two-dimensional space-time representing the $R$-region of the Schwarzschild manifold, $-\infty<t<+\infty$, $-\infty<r^{\star}<+\infty$, i.e., this is exactly that diagram shown in Fig. 1. The null geodesics are straight lines with the slope $\pm 45^{\circ}$. Some of them come from the past horizon to the future infinity, but where from? Others come from the past infinity to the future horizon, but, further, where to? To make this clear it is convenient to introduce the so called retarded and advanced Finkelstein coordinates.

The retarded Finkelstein coordinates are the retarded time $u$ and radius $r$, where

$$
u=t-r^{\star}, \quad d r^{\star}= \pm \frac{d r}{1-r_{g} / r} .
$$

The Schwarzschild metrics (its 2-dim part) then takes the form

$$
d s_{2}^{2}=\left(1-r_{g} / r\right) d u^{2}+2 d u d r
$$

The retarded geodesics $u=$ const are straight lines with the slope $\left(+45^{\circ}\right)$, while the other null geodesic congruence is described by $u+2 r^{\star}=$ const, this follows immediately from the form of the metrics. It is clear now that in the $R_{+}$-region the parameter $u$ for retarded null geodesics runs from $-\infty$ at the null past infinity to $+\infty$ at the future apparent horizon. We see that, written in this coordinates, there are neither singularity nor degeneracy in the metrics, Eqn. (8), at the Schwarzschild radius, so, we can safely continue it into a $T$-region. What kind of? Moving from $R_{+}$-region back in time along retarded null lines, $u=$ const, we inevitably will come to the $T_{+}$-region, since this is the expanding congruence. In $T$ regions the surfaces of constant radii (and, as a consequence, constant "tortoised" coordinate) become, naturally, space-like, so, in order to avoid any confusions one should substitute $t$ by the radial coordinate $q$ in our definition of the retarded time $u$, and since

$$
\frac{d r}{d r^{\star}}= \pm\left(1-r_{g} / r\right)
$$

then in $T_{+}$-region the sign "_" is appropriate. Therefore, the "tortoised" coordinate now runs from $r^{\star}=0$ at $r=0$ to $+\infty$ at the past apparent horizon $(q=+\infty$, $r^{\star}=+\infty, u=q-r^{\star}=$ const). Since the $T_{+}$-region in our case has the intrinsic space-like boundary at $r=r^{\star}=0$, the corresponding conformal diagram is the right-angle triangle shown in Fig. 2, but rotated in such a way that its hypotenuse appeared at the bottom. (The right angle sides are the parts of future and past horizons crossed in the so-called bifurcation point.) Combining $R_{+^{-}}$ and $T_{+}$-regions, we obtain the part of the Carter-Penrose diagram covered by retarded Finkelstein coordinates. It is shown in Fig. 3 The advanced Finkelstein coordinates, $v$ и $r$, are introduce in an analogous way,

$$
v=t+r^{\star}, \quad d r^{\star}= \pm \frac{d r}{1-r_{g} / r},
$$

and the Schwarzschild metric takes the from

$$
d s_{2}^{2}=\left(1-r_{g} / r\right) d v^{2}-2 d v d r
$$


Null geodesics $v=$ const $=u+2 r^{\star}$ are straight lines with the slope $\left(-45^{\circ}\right)$, the parameter $v$ is changing from $-\infty$ at the past apparent horizon to $+\infty$ at the future null infinity, and the ingoing (contracting) congruence itself starts at the past null infinity $\left(t=-\infty, r^{\star}=+\infty, v=\right.$ const), crosses the future apparent horizon $\left(t=+\infty, r^{\star}=-\infty, v=\right.$ const $)$ and then enters the $T_{-}$-region, where the metrics, Eqn. (9) can be safely continued to. In the $T_{-}$-region we, of course, must make the, now familiar, substitution $t \rightarrow q$ and choose the sign "-" in the definition of the "tortoised" coordinate. The part of the Carter-Penrose diagram covered by the advanced Finkelstein coordinates, is shown in Fig. 4. Combining these two, we arrive at Fig. 5.

But still, the constructed manifold is not geodesically complete. The problem is that in the $T_{+}$-region there exist, apart from the null geodesics $u=$ const, yet another null congruence, mentioned above, $v=u+2 r^{\star}=$ const. On the confirmal diagram it is represented by null lines that start at the past singularity $r=0$ and go from right to left with the slope $\left(-45^{\circ}\right)$. They "encounter" the future apparent horizon and can be continued, evidently, further. Where to? Analogously, in the $T_{-}$-region, apart from the already considered null geodesics, $v=$ const, there exist also null geodesics $u=v-2 r^{\star}=$ const. They started at the past apparent horizon and "encounter" the future singularity at $r=0$. Where they come from? It follows from the continuity of the invariant $\Delta$ that there should exist the $R$-region isometric to our $R_{+}$-region we started with (the isometry is the consequence of the Birkhoff theorem [15]). Since, in our construction it should lie to the left of the $T$-regions, i.e., its future and past null infinities are located to the left of the apparent horizons, then the radii should decrease from left to right, and this is, due to our conventions, is the $R_{-}$-region. The difference of the Finkelstein coordinates used before in the $R_{+}$-region is that in the definition of the "tortoised" coordinate $r^{\star}$ one should change the signs "+" $\rightarrow$ "-". The full Carter-Penrose conformal diagram is shown in Fig. 6. Now the obtained manifold is indeed geodesically complete: its boundaries, apart from all the infinities, are two space-like singular surfaces $r=0$ in the past and in the future. They are singular because the Riemann curvature tensor is divergent there. Physically in means that in the vicinity of the surfaces of zero radius the tidal forces grow unboundedly, and nothing can survive.

4.4. On the Causal Structure of the Schwarzschild Manifold. The space-time shown on the above diagram is called "eternal black hole". It was stated in Introduction that the black hole is the part of the space-time beyond the event horizon. And the event horizon is defined as the null hyper-surface, separating the regions where from the light can escape the infinity and those where from it is impossible to do. In such a definition the global property is hidden-to determine the event horizon one should know the whole history, both past and future. The apparent horizon, on the contrary, is defined locally-by zero value of the invariant $\Delta$. In our case the apparent horizon is null hyper-surface, and it is seen clearly on the full conformal diagram that it coincides with the event horizon.

For the generic black hole formed during the gravitational collapse, the picture will be different. Somewhere in the $R_{+}$region there will be a (time-like) boundary of the collapsing matter, that crosses the horizon and comes into the $T_{-}$-region, forming the black hole outside. Consequently, to the left of this boundary the 
Carter-Penrose diagram should be changed. And what serves as a source of gravity (and space-time curvature) in the case of eternal black hole? Naturally, nothing but singularity at $R=0$. Evidently, this source is very strange. We used to think of point-like particle at the center as "staying at one and the same place", i.e., having a time-like world line. Here, instead, we are dealing with two space-like surfaces at $R=0$, in the past and in the future. If the radius is chosen as a temporal coordinate, one would say that the source existed in the past for only one moment, then disappeared and resurrected again, like Phoenix, in the future singularity. Since in the $T$-regions the space-time is essentially non-static, the processes of matter creation can take place, therefore, the situation is unstable. The instability in the $T$-region of inevitable contraction does not disturb us seriously since all the information remains there and finally disappears in the future singularity, not penetrating neither into $R_{--}$nor into $R_{+}$-region (principle of causality!); this is seen rather well in Fig. 6. It is because of this that the $T_{-}$-region is called the black hole. The very name was invented by John Archibald Wheeler [16-18]. The situation in the $T_{+}$-region is quite different. As it seen on the diagram, the created (due to instability) matter can travel both to the $R_{+_{-}}$and to the $R_{-}$-region. In the $R_{+}$-region (where the observers are situated) this will be seen as a bright flash. And this is because the $T_{+}$-region was called the white hole. The part of matter that passed to the $R_{-}$-region (forever!) forms the so called semi-closed world, that shows itself in outer $R_{+}$-region as the gravitating mass in the empty space-time with the hole in it-sphere of the radius equal $R_{g}$.

We have already mentioned that the points in $R_{+}$and in $R_{-}$-regions are causally disconnected, the interval between them is space-like. If we cut the diagram along the space-like hyper-surface that goes through the intersection of past and future horizons, then the geometry of this hyper-surface is called the Einstein-Rosen bridge [19] or non-traversable wormhole. We see, that the causal structure of the geodesically complete Schwarzschild space-time is rather complex, unusual and curious.

\section{Thin Shells}

5.1. Israel Equations. In this Section we present the thin shell technique in General Relativity, elaborated by W. Israel [20-22].

Consider a $d$-dimensional space-time $\mathcal{M}$, divided in two parts, $\mathcal{M}_{-}$и $\mathcal{M}_{+}$ by some hyper-surface $\Sigma$. The hyper-surface $\Sigma$ is called singular, if the energymomentum tensor, concentrated on it has the form

$$
T_{i}^{k}=S_{i}^{k} \delta(\Sigma)+\ldots,
$$

here $S_{i}^{k}$ is the surface energy-momentum tensor of the shell, and $(i, k=0,2, \ldots$, $d-2$ ), otherwise the shell is called nonsingular.

In order to derive the equations of motion for a singular shell, it is necessary, first of all, to construct the metrics on $\mathcal{M}$, continuous while crossing $\Sigma$. Let in some coordinate system $y^{+}$on $\mathcal{M}_{+}$the equation describing the hyper-surface is $F^{+}\left(y^{+}\right)=0$, and, correspondingly, $F^{-}\left(y^{-}\right)=0$ in $\mathcal{M}_{-}$. Then, using the gauge freedom, we can always write the metrics in $\mathcal{M}_{-}$and $\mathcal{M}_{+}$as follows

$$
\begin{aligned}
& d s^{2}=\varepsilon d n^{+2}+\gamma_{i j}^{+}\left(x^{+}, n^{+}\right) d x^{+i} d x^{+j}, \\
& d s^{2}=\varepsilon d n^{-2}+\gamma_{i j}^{-}\left(x^{-}, n^{-}\right) d x^{-i} d x^{-j} .
\end{aligned}
$$


Here

$$
\varepsilon= \begin{cases}-1, & \text { if } \Sigma \text { space-like } \\ +1, & \text { if } \Sigma \text { time-like }\end{cases}
$$

In this new coordinates, $\left(n^{ \pm}, x^{ \pm}\right)$the surface equations $F^{ \pm}\left(y^{ \pm}\right)=0$ become $n^{+}=n^{-}=0$. The continuity condition for the metrics on $\mathcal{M}$ is the existence of the coordinate transformation, $x^{+}=x^{+}\left(x^{-}\right)$, such that

$$
\gamma_{i j}^{+}\left(x^{+}, 0\right)=\gamma_{k l}^{+}\left(x^{-}, 0\right) \frac{\partial x^{-k}}{\partial x^{+j}} \frac{\partial x^{-l}}{\partial x^{+i}}
$$

Note, that $\gamma_{k l}\left(x^{-}, 0\right)$ is a $(n-1)-\operatorname{dim}$ tensor. Thus, we are able to choose the following metrics on $\mathcal{M}$ :

$$
d s^{2}=\varepsilon d n^{2}+\gamma_{i j}(x, n) d x^{i} d x^{j}
$$

This metrics is not however smooth, its first derivatives in the normal coordinate $n$ can jump on $\Sigma$. Since the metrics, Eqn. (10), gives us the normal slicing on $\mathcal{M}$, one can use the Gauss-Codazzi relations and rewrite the Einstein equations in terms of extrinsic curvature tensor $K_{i j}$ and scalar curvature ${ }^{(d-1)} R$ of the $(d-1)$-dim normal section. Then the components $\left(\begin{array}{l}i \\ n\end{array}\right)$ and $\left(\begin{array}{l}n \\ n\end{array}\right)$ become

$$
\begin{gathered}
G_{i}^{n}=K_{i \mid j}^{j}-K_{l \mid i}^{l}=-8 \pi G T_{i}^{n}, \\
G_{n}^{n}=-\frac{1}{2}{ }^{(d-1)} R \varepsilon+\frac{1}{2}\left((\operatorname{Tr} K)^{2}-\operatorname{Tr} K^{2}\right)=8 \pi G T_{n}^{n}, \\
G_{i}^{j}={ }^{(d-1)} G_{i}^{j}+\varepsilon\left(K_{i}^{j}-\delta_{i}^{j} \operatorname{Tr} K\right), n-\varepsilon(\operatorname{Tr} K) K_{i}^{j}+ \\
+\frac{1}{2} \varepsilon \delta_{i}^{j}\left\{(\operatorname{Tr} K)^{2}+\operatorname{Tr} K^{2}\right\}=8 \pi G T_{i}^{j} .
\end{gathered}
$$

Here "|" denotes the covariant derivative with respect to the metrics on $(d-1)$-dim normal section. In gaussian coordinates one has

$$
K_{i}^{j} \equiv \Gamma_{i n}^{j}=-\frac{1}{2} \gamma^{j k} \gamma_{k i, n}
$$

and in the case of jump in the first derivatives, the second term in Eqn. (13) will contain a $\delta$-function. After integrating the Eqn. (13) in $n$,

$$
\lim _{\delta \rightarrow 0}\left[\int_{-\delta}^{\delta} G_{i}^{j} d n\right]=8 \pi G \lim _{\delta \rightarrow 0}\left[\int_{-\delta}^{\delta} T_{i}^{j} d n\right],
$$

one gets the equation of motion for the shell:

$$
\varepsilon\left(\left[K_{i}^{j}\right]-\delta_{i}^{j}\left[K_{l}^{l}\right]\right)=8 \pi G S_{i}^{j}
$$

where $\left[K_{i}^{j}\right] \equiv K_{i}^{j^{+}}-K_{i}^{j-}$ is the jump in the extrinsic curvature tensor.

Making use of Eqn. (14), one gets from Eqs. (12) and (11)

$$
S_{i \mid j}^{j}+\left[T_{i}^{n}\right]=0
$$




$$
\left\{K_{i}^{j}\right\} S_{j}^{i}+\left[T_{n}^{n}\right]=0
$$

where

$$
\left\{K_{i}^{j}\right\} \equiv \frac{1}{2}\left(K_{i}^{j^{+}}+K_{i}^{j^{-}}\right) .
$$

Eqs. (15) and (16) are the continuity equations for the surface energy-momentum tensor of the shell.

The above derivation of Israel equations are based essentially on the fact that the shell $\Sigma$ is singular. However, one can also obtain them as a consequence of the Einstein equations written on the manifold decomposed in $\mathcal{M}, \mathcal{M}_{+}, \mathcal{M}_{-}$and $\Sigma$. Then, in addition to the Israel equations there appear as well equations valid everywhere in the space-time. The energy-momentum tensor in this case can be written as

$$
T_{\mu \nu}(n, x)=S_{\mu \nu}(n, x) \delta(n)+E_{\mu \nu}(n, x) \theta(n)+\tilde{T}^{-}{ }_{\mu \nu}(n, x),
$$

where

$$
\left.\left[\tilde{T}_{\mu \nu}\right]\right|_{n=0}=0, \quad \tilde{T}^{+}{ }_{\mu \nu}=E_{\mu \nu}+\tilde{T}^{-}{ }_{\mu \nu} .
$$

Let us now write the extrinsic curvature tensor as a pair $\left(K_{+}, K_{-}\right)$, where $K_{ \pm}$ are extrinsic curvatures of $\Sigma$ in the regions $\mathcal{M}_{ \pm}$:

$$
K_{i j}{ }^{+}=\left\{K_{i j}\right\}+\frac{1}{2}\left[K_{i j}\right], \quad K_{i j}{ }^{-}=\left\{K_{i j}\right\}-\frac{1}{2}\left[K_{i j}\right] .
$$

As was already shown, in the case of singular shell the derivatives of metric tensor undergo a jump when crossing the shell, and, consequently, the jump in the extrinsic curvature tensor is nonzero, $\left[K_{i j}\right] \neq 0$. When the shell is nonsingular, then $S_{\mu \nu}=0$ and $\left[K_{i j}\right]=0$. However, the derivative of the extrinsic curvature tensor along the normal direction may undergo a jump, $\left[K_{i j}\right],_{n} \neq 0$. In this case we are dealing with the shock waves.

Keeping in mind this note, we can write the extrinsic curvature tensor and its normal derivative in the form

$$
\begin{gathered}
K_{i j}(n, x)=\left[K_{i j}(n, x)\right] \theta(n)+K^{-}{ }_{i j}(n, x), \\
K_{i j}(n, x){ }_{n}=\left[K_{i j}(n, x)\right] \delta(n)+\left[K_{i j}(n, x)\right],{ }_{n} \theta(n)+K^{-}{ }_{i j}(n, x) .
\end{gathered}
$$

Note that functions $K^{-}{ }_{i j}$ are considered as continuous everywhere, $-\infty<n<+\infty$. Substituting these relations into the left hand sides of the Einstein's equations and equating the coefficients in front of the corresponding $\delta$-functions, one arrives at the Israel equations.

To be more detailed, let us write the Einstein equations by components, using Eqn. (18):

$$
\begin{aligned}
& G_{n n}=\left(-\left[K_{i}^{j}\right]\left\{K_{j}^{i}\right\}+[\operatorname{Tr} K]\{\operatorname{Tr} K\}\right) \theta(n)-K_{i}^{-j} K^{-i}{ }_{j}-\left(\operatorname{Tr} K^{-}\right)^{2}-\frac{1^{(d-1)}}{2} R \varepsilon \\
& \begin{array}{r}
G_{n i}=\left(-\left[K_{i \mid l}^{l}\right]+\operatorname{Tr} K_{\mid i}\right) \theta(n)+\left(-K^{-l}{ }_{i \mid l}+\operatorname{Tr} K^{-} \mid i\right) \\
\quad=8 \pi G \varepsilon S_{i \mid l}^{l} \theta(n)+\left(-K^{-l}{ }_{i \mid l}+\operatorname{Tr} K^{-}{ }_{\mid i}\right)
\end{array}
\end{aligned}
$$




$$
\begin{gathered}
G_{i j}=\varepsilon\left(\left[K_{i j}\right]-\gamma_{i j}[\operatorname{Tr} K]\right) \delta(n)+\varepsilon\left(\left[K_{i j}, n\right]-\gamma_{i j}\left[\operatorname{Tr} K,_{n}\right]\right) \theta(n)+ \\
+8 \pi G\left(2 S_{i}^{l}\left\{K_{l j}\right\}+2 S_{j}^{l}\left\{K_{l i}\right\}-\frac{3}{d-2} \operatorname{Tr} S\left\{K_{i j}\right\}-S_{i j}\{\operatorname{Tr} K\}\right) \theta(n)+ \\
+8 \pi G\left(\gamma_{i j} S_{m}^{l}\left\{K_{l}^{m}\right\}-\frac{1}{d-2} \gamma_{i j} \operatorname{Tr} S\{\operatorname{Tr} K\}\right) \theta(n)+ \\
+\varepsilon\left(K_{i j}^{-},{ }_{n}-\gamma_{i j} \operatorname{Tr} K^{-}{ }_{n}+2 K^{-}{ }_{i l} K^{-l}{ }_{j}-K^{-}{ }_{i j} \operatorname{Tr} K^{-}\right)+ \\
+\frac{1}{2} \varepsilon \gamma_{i j}\left(K^{+l}{ }_{m} K^{-m}+\operatorname{Tr} K^{-2}\right)+{ }^{(d-1)} G_{i j} .
\end{gathered}
$$

Equating these components to the corresponding ones for the energy-momentum tensor, Eqn. (17), one obtains

$$
\begin{gathered}
\left\{K_{i}^{j}\right\} S_{j}^{i}+\left[T_{n}^{n}\right]=0 \\
-K_{i}^{-j} K^{-i}{ }_{j}-\left(\operatorname{Tr} K^{-}\right)^{2}-\frac{1}{2}(d-1) \operatorname{Re}=8 \pi G \tilde{T}_{n n} ; \\
S_{i \mid j}^{j}+\left[T_{i}^{n}\right]=0 \\
-K^{-l}{ }_{i \mid l}+\operatorname{Tr} K^{-}{ }_{\mid i}=8 \pi G \tilde{T}_{n i} ; \\
\varepsilon\left(\left[K_{i}^{j}\right]-\delta_{i}^{j}\left[K_{l}^{l}\right]\right)=8 \pi G S_{i}^{j}, \\
2 S_{i}^{l}\left\{K_{l j}\right\}+2 S_{j}^{l}\left\{K_{l i}\right\}-\frac{3}{d-2} \operatorname{Tr} S\left\{K_{i j}\right\}-S_{i j}\{\operatorname{Tr} K\}+ \\
+\gamma_{i j} S_{m}^{l}\left\{K_{l}^{m}\right\}-\frac{1}{d-2} \gamma_{i j} \operatorname{Tr} S\{\operatorname{Tr} K\}=\left[T_{i j}\right], \\
\varepsilon\left(K^{-}{ }_{i j},{ }_{n}-\gamma_{i j} \operatorname{Tr} K^{-}{ }_{n}+2 K^{-}{ }_{i l} K^{-l}{ }_{j}-K^{-}{ }_{i j} \operatorname{Tr} K^{-}+\right. \\
\left.+\frac{1}{2} \gamma_{i j}\left(K^{+l}{ }_{m} K^{-m}{ }_{l}+\operatorname{Tr} K^{-2}\right)\right)+{ }^{(d-1)} G_{i j}=8 \pi G \tilde{T}_{i j} .
\end{gathered}
$$

It can be easily seen, that the first lines, Eqs. (19), (20) and (21), are exactly the Israel equations. They are non-trivial if the shell is singular. In the case of shock waves they serve as the matching conditions for different types of the metrics. The remaining six equations are non-trivial everywhere in the space-time. In the absence both the singular shells and the shock waves these equations are simply the " $(d+1)$-decomposition" of the Einstein equations.

5.2. Spherically Symmetric Thin Shells. In what follows we will only be dealing with the spherically symmetric thin shells in "our" four-dimensional space-time. So, we will study this case in details following he work [11].

Let us write the spherically symmetric metrics in already diagonal form

$$
d s^{2}=e^{\nu} d t^{2}-e^{\lambda} d q^{2}-r^{2}(t, q)\left(d \vartheta^{2}+\sin ^{2} \vartheta d \varphi^{2}\right) .
$$

In the Gaussian normal coordinate system, related to the shell, $e^{\lambda}=1, q=n$, and we introduce the proper time $\tau$ for the observer sitting on this shell at $n=0$ : 
$d \tau=e^{\frac{\nu}{2}(t, 0)} d t$. Denoting $\rho(\tau)=R(t, 0)$, we get for the invariant $\Delta$ :

$$
\Delta=\dot{\rho}^{2}-\rho_{n}^{2}
$$

where "dot" means differentiation with respect to the proper time $\tau$, and lower index $n$-differentiation along he normal to the shell. Because of spherical symmetry and diagonal structure of the metrics, the surface energy-momentum tensor of the shell is very simple:

$$
S_{i}^{j}=\operatorname{diag}\left(S_{0}^{0}, S_{2}^{2}, S_{2}^{2}\right)
$$

The same structure is for the extrinsic curvature tensor $K_{i}^{j}$. So, of all the matching equations there remain only two Israel equations and the continuity equation that now read as

$$
\begin{gathered}
{\left[K_{2}^{2}\right]=4 \pi G S_{0}^{0},} \\
{\left[K_{0}^{0}\right]+\left[K_{2}^{2}\right]=8 \pi G S_{2}^{2},} \\
\left\{K_{0}^{0}\right\} S_{0}^{0}+(d-2)\left\{K_{2}^{2}\right\} S_{2}^{2}+\left[T_{n}^{n}\right]=0, \\
\dot{S}_{0}^{0}+2 \frac{\dot{\rho}}{\rho}\left(S_{0}^{0}-S_{2}^{2}\right)+\left[T_{0}^{n}\right]=0,
\end{gathered}
$$

the continuity equation being a differential consequence of the Israel equations and the Einstein equations on the shell. It is easy to see that $K_{2}^{2}=-\rho, n / \rho$; $K_{0}^{0}=-\nu,{ }_{n} / 2$. Remembering now, how the invariant $\Delta$ looks like, we get:

$$
\rho_{n}= \pm \sqrt{\dot{\rho}^{2}-\Delta}=\sigma \sqrt{\dot{\rho}^{2}-\Delta}
$$

Here there appeared the sign function $\sigma= \pm 1$, that shows, whether the radii increase in direction of the outer normal, or they decrease. We know already that in the $R$-regions the sign of the spatial derivative of the radius does not change. This means that the value of $\sigma$ determines in which of them the shell is traveling: if this happens in $R_{+}$- then $\sigma=+1$, if in $R_{-}$- then $\sigma=-1$. In general, $\sigma$ is not an integral of motion and may change its value in $T$-regions. Since we will be dealing with the shells in vacuum, i.e., both inside and outside of which are Schwarzschild space-times with different mass parameters, the signs of $\sigma_{\text {in }}$ inside and $\sigma_{\text {out }}$ outside determine, in what manner these two geometries are matched along the shell, i.e., they define completely the global geometry of the full manifold. In the simplest case, when there is the flat Minkowski space-time inside, $\sigma_{\text {in }}=+1$ and the sign of $\sigma_{\text {out }}$ defines the type of shell: black hole for $\sigma_{\text {out }}=+1$ or wormhole (semiclosed world) for $\sigma_{\text {out }}=-1$.

Let us start with calculation of extrinsic curvature tensor on the shell. Since, locally, the geometry of the spherically symmetric space-time is completely determined by two invariant functions, $R(t, q)$ и $\Delta(t, q)$, it is clear that the result should depend on the values of $\rho(\tau)$ and $\Delta(\tau)$ and their time derivatives. For the jump of $\left[K_{2}^{2}\right]$, we have, evidently,

$$
\left[K_{2}^{2}\right]=-\left[\frac{\sigma}{\rho} \sqrt{\dot{\rho}^{2}-\Delta}\right]
$$


The jump of $\left[K_{0}^{0}\right]$ can also be calculated straightforwardly, but it is easier to make use of the first of the Israel equations, continuity equation and the Einstein equations in the vectorial form derived in the preceding Section. So, we have:

$$
\begin{gathered}
-\left[\sigma \sqrt{\dot{\rho}^{2}-\Delta}\right]=4 \pi G \rho S_{0}^{0}, \\
\dot{S}_{0}^{0}+\frac{2 \dot{\rho}}{\rho}\left(S_{0}^{0}-S_{2}^{2}\right)+\left[T_{0}^{n}\right]=0, \\
(\rho(1+\Delta))_{i}=8 \pi G \rho^{2}\left(T \rho,_{i}-T_{i}^{k} \rho,{ }_{k}\right) .
\end{gathered}
$$

Putting $i=0$ in the last of these equations, we get

$$
\dot{\rho}(1+\Delta)+\rho \dot{\Delta}=8 \pi G \rho^{2}\left(T_{n}^{n} \dot{\rho}-T_{0}^{n} \rho_{n}\right) .
$$

Let us differentiate the first equation

$$
-\left[\frac{\sigma}{2 \sqrt{\dot{\rho}^{2}-\Delta}}(2 \ddot{\rho}-\dot{\Delta})\right]=4 \pi G \dot{\rho} S_{0}^{0}+4 \pi G \rho \dot{S}_{0}^{0}
$$

and substitute into it the expression for $\dot{S}_{0}^{0}$ from the continuity equation:

$$
\left[\frac{\sigma}{2 \sqrt{\dot{\rho}^{2}-\Delta}}(2 \ddot{\rho}-\dot{\Delta})\right]=4 \pi G\left(2 S_{2}^{2}-S_{0}^{0}\right) \dot{\rho}+4 \pi G \rho\left[T_{0}^{n}\right] .
$$

The only thing left is to substitute in here the expression for $\dot{\Delta}$ from Einstein equations and divide the whole relation by $\dot{\rho}$. The result is the following

$$
-\left[\frac{\sigma}{2 \sqrt{\dot{\rho}^{2}-\Delta}}\left(\ddot{\rho}+\frac{1+\Delta}{2 \rho}-4 \pi G \rho T_{n}^{n}\right)\right]=4 \pi G\left(2 S_{2}^{2}-S_{0}^{0}\right) .
$$

Finally, the system of Israel equations for matching geometries inside and outside the shell and defining its evolution plus the continuity equation for the surface energy-momentum tensor take the form

$$
\begin{gathered}
-\left[\sigma \sqrt{\dot{\rho}^{2}-\Delta}\right]=4 \pi G \rho S_{0}^{0}, \\
-\left[\frac{\sigma}{\sqrt{\dot{\rho}^{2}-\Delta}}\left(\ddot{\rho}+\frac{1+\Delta}{2 \rho}-4 \pi G \rho T_{n}^{n}\right)\right]=4 \pi G\left(2 S_{2}^{2}-S_{0}^{0}\right), \\
\dot{S}_{0}^{0}+\frac{2 \dot{\rho}}{\rho}\left(S_{0}^{0}-S_{2}^{2}\right)+\left[T_{0}^{n}\right]=0 .
\end{gathered}
$$

The structure of these equations reflects the general structure of the Einstein equations. The first is the equation of the initial conditions or, using the language of Hamiltonian formalism, the constraint equation. The second equation is dynamical-it contains the second time derivative-acceleration. The third one is the consequence of the Bianchi identities. The invariant functions $\Delta$ (in, out), and also $T_{0}^{n}$ (in, out) and $T_{n}^{n}$ (in, out) are determined by the properties of the inner and outer parts of the space-time and the matter distributions there. So, we have two equations for three unknown functions of proper time, namely, $\rho(\tau), S_{0}^{0}(\tau)$ 
and $S_{2}^{2}(\tau)$. As was already mentioned, we study he shell placed in the vacuum, therefore, $T_{0}^{n}=0$ и $T_{n}^{n}=0$. Besides, for the Schwarzschild solution we have

$$
\Delta=1-2 G m / \rho,
$$

where inside $m=m_{\text {in }}$, outside $m=m_{\text {out }}$.

5.3. Initial Condition and Dynamics. Let us now decide what kind of shells we want to study. We are choosing the simplest generalization of the point-like gravitating source, namely, the spherically symmetric thin dust shell. The word "dust" means that its tangential stress is zero, $S_{2}^{2}=0$. Let $M$ be the sum of all the masses of particles composing the shell. It is called the "bare mass". In the chosen units $M=E$-i.e., this is the energy of the shell in its hydrodynamical or thermodynamical meaning. By definition, it equals to the following volume integral:

$$
\begin{gathered}
M=\int T_{0}^{0} d V=4 \pi \int T_{0}^{0} R^{2} e^{\frac{\lambda}{2}} d q=4 \pi \int S_{0}^{0} \delta(n) R^{2} d n=4 \pi \rho^{2}(\tau) S_{0}^{0}, \\
S_{0}^{0}=\frac{M}{4 \pi \rho^{2}} .
\end{gathered}
$$

After substituting this into the continuity equation one gets, that for the dust shell $M=$ const, i.e., the bare mass is the integral of motion. So, we are left with only one unknown function, the radius of the shell $\rho(\tau)$. Of course, it can be found by integrating the dynamical equation. The general solution will depend on two arbitrary constants, which can be specified by imposing initial conditions $\rho_{0}=\rho(0), \dot{\rho}_{0}=\dot{\rho}(0), \sigma_{\text {in }}(0)$ and $\sigma_{\text {out }}(0)$, provide that all the other parameters, Schwarzschild masses $m_{\text {in }}, m_{\text {out }}$ and the bare mass $M$ are known. But, in addition, we have also the first equation, that constraints the set of initial conditions and the values of parameters. This can be demonstrated in the following way: let $\rho_{0}$, $\dot{\rho}_{0}, m_{\text {in }}$ and $M$ be given, then, from the constraint equation for different values of $\sigma_{\text {in }}(0)$ and $\sigma_{\text {out }}(0)$ we would obtain different values for $m_{\text {out }}$, i.e., for the total mass (energy) of the system accounting for the gravitational mass defect. And, vice verse, specifying from the very beginning the parameters of the system, $m_{\text {in }}$, $m_{\text {out }}$ and $M$, we will be able (after some investigation) to obtain the possible values for $\sigma_{\text {in }}(0)$ and $\sigma_{\text {out }}(0)$, defining, in this way, the global geometry of the complete space-time. For this it sufficient to know only one constraint differential equation, which is of the first order. Such an investigation we will start right now.

Let us write this equation in the explicit form:

$$
\sigma_{\text {in } \sqrt{\dot{\rho}^{2}+1-\frac{2 G m_{\text {in }}}{\rho}}}-\sigma_{\text {out }} \sqrt{\dot{\rho}^{2}+1-\frac{2 G m_{\text {out }}}{\rho}}=\frac{G M}{\rho} .
$$

We assume that the shell consists of an ordinary, not exotic, matter, i.e., let $M>0$. Since we are interested in black holes, we consider only the case when $m_{\text {out }}>0$. We do not need to integrate our differential equation, it is only sufficient to investigate some specific points of the trajectories, namely, $\rho=0, \infty$, turning points $\dot{\rho}=0$ as well as signs of $\sigma_{\text {in }}, \sigma_{\text {out }}$ there. Therefore, we will proceed in the following way. First, let us make a square of the equation after moving the second 
term from the left to right,

$$
\frac{\Delta m}{M}=\sigma_{\text {out }} \sqrt{\dot{\rho}^{2}+1-\frac{2 G}{\rho}}+\frac{G M}{2 \rho},
$$

where $\Delta m=m_{\text {out }}-m_{\text {in }}$ is the total mass of the shell. It follows from this that

$$
\sigma_{\text {out }}=\operatorname{sign}\left(\frac{\Delta m}{M}-\frac{G M}{2 \rho}\right),
$$

so,

$$
\sigma_{\text {out }}(0)=-1, \quad \sigma_{\text {out }}(\infty)=\operatorname{sign}\left(\frac{\Delta m}{M}\right)
$$

Note, that nothing forbids the value of $\Delta m$ to be negative for $m_{\text {in }}>0$. For the sign of $\sigma_{\text {in }}$ one has

$$
\sigma_{\text {in }}=\operatorname{sign}\left(\frac{\Delta m}{M}+\frac{G M}{2 \rho}\right)
$$

so,

$$
\sigma_{\text {in }}(0)=+1, \quad \sigma_{\text {in }}(\infty)=\operatorname{sign}\left(\frac{\Delta m}{M}\right)
$$

When the shell's motion is infinite (unbounded), a when it is finite (bounded)? Letting the radius of the shell to go to infinity, one gets

$$
\frac{\Delta m}{M}=\sigma_{\text {out }} \sqrt{\dot{\rho}^{2}+1}=\sigma_{\text {in }} \sqrt{\dot{\rho}^{2}+1},
$$

therefore, the motion is infinite if $|\Delta m| / M \geqslant 1$, and in this case $\sigma_{\text {in }}(\infty)=$ $=\sigma_{\text {out }}(\infty)$. If $|\Delta m| / M \leqslant 1$, then the motion is finite.

For the turning points, $\dot{\rho}=0$, one obtains simple quadratic equation:

$$
\left(\frac{G M}{2 \rho_{0}}\right)^{2}+2 \frac{m_{\text {in }}+m_{\text {out }}}{M}\left(\frac{G M}{2 \rho_{0}}\right)+\left(\frac{\Delta m}{M}\right)^{2}-1=0 .
$$

It is easy to see that for the infinite motion there are no turning points (both roots of the quadratic equation either negative or complex conjugate), and in the case of finite motion there exists only one turning point $\rho=\rho_{0}>0$ :

$$
\frac{2 G M}{\rho_{0}}=-\frac{m_{\mathrm{in}}+m_{\mathrm{out}}}{M}+\sqrt{1+\frac{4 m_{\mathrm{in}} m_{\mathrm{out}}}{M^{2}}} .
$$

5.4. Global Geometries. Let us consider, first, the infinite motion, which is determined completely by the sign of $\Delta m$. Indeed, $\operatorname{sign} \Delta m=\sigma_{\text {in }}(\infty)=\sigma_{\text {out }}(\infty)$, and it is always $\sigma_{\text {in }}(0)=+1$ и $\sigma_{\text {out }}(0)=-1$. If $\Delta m>0$, then the CarterPenrose diagram for the whole space-time looks like the following, see Fig. 7. The trajectory is rather schematic: we did not show explicitly the changes of $\sigma$ both inside and outside the shell in the vicinity of zero radii. By dashed curves the surfaces of constant radii are indicated in the corresponding $R$ - and $T$-regions. The relative positions of the apparent horizons in the outer and inner metrics 
are chosen such that the condition $\Delta m>0$ is fulfilled: the shell when contracting from the infinite radius to zero, crosses first the outer horizon and enters the outer $T_{ \pm \text {out }}$-region, and only then-inner one. Of course, there exists also the solution with the expanding shell, which can be obtained simply by time reversion. In the case when $\Delta m<0$, the Carter-Penrose diagram is shown in Fig. 8

Consider now the finite motion, which provides us with much more different global geometries. Thus, what is at hand? Substituting the value of $\rho_{0}$, found before, into the expression for $\sigma$, we obtain the following inequalities:

$$
\frac{\Delta m}{M}>\frac{1}{2}\left(\sqrt{1+\frac{m_{\text {in }}^{2}}{M^{2}}}-\frac{m_{\text {in }}}{M}\right) ; \quad \frac{\Delta m}{M}<-\frac{1}{2}\left(\sqrt{1+\frac{m_{\text {in }}^{2}}{M^{2}}}+\frac{m_{\text {in }}}{M}\right),
$$

if $\sigma_{\text {out }}=+1$, and

$$
-\frac{1}{2}\left(\sqrt{1+\frac{m_{\text {in }}^{2}}{M^{2}}}+\frac{m_{\text {in }}}{M}\right)<\frac{\Delta m}{M}<\frac{1}{2}\left(\sqrt{1+\frac{m_{\text {in }}^{2}}{M^{2}}}-\frac{m_{\text {in }}}{M}\right),
$$

if $\sigma_{\text {out }}=-1$. Analogously, for inner metrics we get:

$$
\frac{\Delta m}{M}>-\frac{M}{4 m_{\text {in }}},
$$

if $\sigma_{\text {in }}=+1$, and

$$
\frac{\Delta m}{M}<-\frac{M}{4 m_{\text {in }}},
$$

if $\sigma_{\text {in }}=-1$. What are the possibilities? When $\Delta m>0$, then $\sigma_{\text {in }}=+1$, and for

$$
\frac{\Delta m}{M}>\frac{1}{2}\left(\sqrt{1+\frac{m_{\text {in }}^{2}}{M^{2}}}-\frac{m_{\text {in }}}{M}\right)
$$

one has $\sigma_{\text {out }}\left(\rho_{0}\right)=+1$, the conformal Carter-Penrose diagram looks as follows, see Fig. 9.

When

$$
0<\frac{\Delta m}{M}<\frac{1}{2}\left(\sqrt{1+\frac{m_{\text {in }}^{2}}{M^{2}}}-\frac{m_{\text {in }}}{M}\right),
$$

then $\sigma_{\text {out }}=-1$ and Carter-Penrose diagram looks as follows, see Fig. 10 .

In the case when $\Delta m<0$, it is always $\sigma_{\text {out }}=-1,(M>0 !)$, and for

$$
-\frac{M}{4 m_{\text {in }}}<\frac{\Delta m}{M}<0
$$

one has $\sigma_{\text {in }}\left(\rho_{0}\right)=+1$, the Carter-Penrose diagram looks as follows, see Fig. 11 .

When

$$
\frac{\Delta m}{M}<0,
$$

then $\sigma_{\text {in }}\left(\rho_{0}\right)=-1$, but this is possible only if

$$
\frac{m_{\text {in }}}{M}>\frac{1}{2 \sqrt{2}} \quad(M>0 !) .
$$


The Carter-Penrose diagram in this case is shown in Fig. 12.

5.5. Modified Newton's Law. We have already said above that in the case of spherical symmetry, having at hand the equation of state for the matter on the shell and after integrating the continuity equation, it is sufficient to investigate the remained of Israel equations, namely, the constraint one, in order to describe, qualitatively, the global geometry of the space-times. However, though the constraint equation is, in a essence, the energy conservation law, its conventional parts - kinetic and potential ones - are not expressed as explicit as one would wish. That's why we decided to derive in this Section, in order to make the physics more clear, the modified Newton's law, i.e., the formula for the acceleration of freely falling spherically symmetric thin dust shell which is the simplest generalization of point-like particle in the central gravitational field.

The Israel equations for the spherically symmetric dust shell have the form

$$
\begin{aligned}
& \sigma_{\text {in }} \sqrt{\dot{\rho}^{2}+1-\frac{2 G m_{\text {in }}}{\rho}}-\sigma_{\text {out }} \sqrt{\dot{\rho}^{2}+1-\frac{2 G m_{\text {out }}}{\rho}}=\frac{G M}{\rho}, \\
& \frac{\sigma_{\text {in }}}{\sqrt{\dot{\rho}^{2}+1-\frac{2 G m_{\text {in }}}{\rho}}}\left(\ddot{\rho}+\frac{G m_{\text {in }}}{\rho^{2}}\right)-\frac{\sigma_{\text {out }}}{\sqrt{\dot{\rho}^{2}+1-\frac{2 G m_{\text {out }}}{\rho}}}\left(\ddot{\rho}+\frac{G m_{\text {out }}}{\rho^{2}}\right)=-\frac{G M}{\rho^{2}},
\end{aligned}
$$

where $m_{\text {in }}$ is the central gravitating mass, and $m_{\text {out }}=m_{\text {in }}+\Delta m$ is the total mass (energy) of the system. Squaring the first equation we get

$$
\sigma_{\text {in }} \sqrt{\dot{\rho}^{2}+1-\frac{2 G m_{\text {in }}}{\rho}}=\frac{\Delta m}{M}+\frac{G M}{2 \rho}, \quad \sigma_{\text {out }} \sqrt{\dot{\rho}^{2}+1-\frac{2 G m_{\text {out }}}{\rho}}=\frac{\Delta m}{M}-\frac{G M}{2 \rho} .
$$

Further, rather simple algebraic calculations give the following result

$$
\ddot{\rho}=-\frac{G\left(m_{\text {in }}+\frac{1}{2} \Delta m\right)}{\rho^{2}}-\frac{G^{2} M^{2}}{4 \rho^{3}} .
$$

The first term in the right hand side is the classical Newton's law for the free fall acceleration (in terms of the proper time of the co-moving observer), but a little bit different: instead of the central gravitating mass $m_{\text {in }}$ we have now

$$
m_{\text {in }}+\frac{1}{2} \Delta m=\frac{1}{2}\left(m_{\text {in }}+m_{\text {out }}\right),
$$

i.e., one half of the Schwarzschild masses inside and outside of the shell. It is not, however, as primitive as it seems. The main thing is that the total mass of the system $m_{\text {out }}$, and, consequently, $\Delta m$ as well, depend on the initial conditions of the shell's motion. In other words, such an additional quantity $\frac{1}{2} \Delta m$ depends on the velocity at the moment of measurement, i.e., on the kinetic energy, what, of course, in the spirit of the main General Relativity postulate: "any kind of energy gravitates".

The second term is a result of gravitational self-interaction, it is proportional not to the inverse square of the radius, but to the inverse cube. The term of such a 
type appears also as a special relativistic modification to the Newton's law which causes the shift of planet's perihelia. Taking into account functionally the same yet another additional term may become useful in estimation of the masses of the super-massive black holes in the galactic centers around which there are stars rotating sufficiently close to the black hole horizons. The curious fact is that if $\sigma_{\text {in }}=+1$, and $\sigma_{\text {out }}=-1$, the shell will shrink to zero radius even in the case when there is nothing to be attracted to, i.e., when $m_{\text {in }}=m_{\text {out }}=0(\Delta m=0)$, just due to the self-gravitation.

We should note, that making measurements one can find out these deviations from the Newton's law and define the global geometry of gravitating system "central body + shell", and this is impossible for the limit of the probe particle. Naturally, it is an ideal case, because we consider the spherically symmetric problem without the gravitational radiation. But it's important to show the necessity for taking into account the inverse influence of gravitational field sources on the metrics and global geometry of the space-time.

\section{Vaidya Metrics}

Israel equations "work good" when the shell either time-like of space-like. And what to do if it is null? As is well known, the normal vector to he null hypersurface lies in that very hyper-surface. But, it is always possible to introduce the double-null coordinates, i.e., the retarded time and the advanced time. The null shell moves, by definition, along one of them. By integrating the Einstein equations along the second one we can obtain the matching conditions on the null hyper-surface [11]. The very question about the investigation of singular (when the energy-momentum tensor is proportional to $\delta$-function) null hypersurfaces may seem purely academic. But this is not so. The examples are jumps in the relativistic fields distributions. Their evolution means the existence of the infinite (in absolute value) temporal and spatial derivatives evolving along the characteristics of the corresponding hyperbolic partial differential equation, i.e., with the speed of light-shock waves. But we will not develop such a formalism here, since there exists the general solution to the Einstein equations for the gravitating spherically symmetric radiation, namely, the Vaidya metrics.

In order to derive the Vaidya metrics it is possible, of course, to go the standard way: to investigate the structure of the energy-momentum tensor for the spherically symmetric radiation and then to try to solve the non-linear Einstein equations (e.g., in the vectorial form described above). But, "we will choose a different way". Let us imagine that the radiation (either outgoing or ingoing) lasts for only finite time interval, then, since nothing can travel faster that light, before the beginning as well as after the radiation, we necessary will be dealing with the Schwarzschild metrics (because of the Birkhoff theorem) with different, of course, mass parameters. Therefore, at the boundaries along the null hypersurfaces one must match the Vaidya and Schwarzschild space-times. It is from this matching that we will obtain all the needed information.

As the first step we should choose the appropriate coordinate system, most adequate to our problem. Let us denote the common notation $z=\{u, v\}$ for the retarded, $u$, and advanced, $v$, null coordinates. For the Schwarzschild metrics, as we already know,

$$
z=t-\varepsilon r^{\star}, \quad d r^{\star}=\frac{d r}{F}, \quad F=1-\frac{2 G m}{r},
$$


where $\varepsilon=+1$ for $z=u$ and $\varepsilon=-1$ for $z=v$, and the line element in the Finkelstein coordinates $\{z, r\}$ takes the form

$$
d s^{2}=F d z^{2}+2 \varepsilon d z d r-r^{2}\left(d \vartheta^{2}+\sin ^{2} \vartheta d \varphi^{2}\right) .
$$

In general, the use of the Finkelstein coordinates dictates the following structure for the spherically symmetric metrics

$$
d s^{2}=A(z, r) d z^{2}+2 H d z d r-r^{2}\left(d \vartheta^{2}+\sin ^{2} \vartheta d \varphi^{2}\right),
$$

because when $z=$ const, its two-dimensional part should equal zero. Note also that the choice of the invariant radius $r$ as the second coordinate (that could be either spatial or temporal) fixes the coordinate system up to arbitrary transformation of the form $z^{\prime}=z^{\prime}(z)$. Let $z=z_{0}$ is the boundary between Vaidya and Schwarzschild metrics. Then, it follows form the continuity of the line element $(=$ the first quadratic form) that the radius $r$ should also be a continuous function along the whole boundary. But the second quadratic form-this is our invariant $\Delta$ - should also be continuous, what becomes evident if one integrates the Einstein equations in the vectorial form "across" the boundary $z=z_{0}$ (i.e., along the other null coordinate) and take into account the absence of $\delta$-function in right hand side of the equations. We have, therefore,

$$
\Delta_{\text {Vaidya }}\left(z_{0}\right)=\Delta_{\text {Schw }}\left(z_{0}\right), \quad \Delta_{\text {Vaidya }}=-1+\frac{2 G m(z)}{r} .
$$

The two-dimensional metric tensor $\gamma_{i k}\left(d s^{2}=\gamma_{i k} d x^{i} d x^{k}=A d z^{2}+2 H d z d r\right)$ and its reverse $\gamma^{i k}$ are

$$
\gamma_{i k}=\left(\begin{array}{cc}
A & H \\
H & 0
\end{array}\right), \quad \gamma^{i k}=\left(\begin{array}{cc}
0 & \frac{1}{H} \\
\frac{1}{H} & -\frac{A}{H^{2}}
\end{array}\right) .
$$

From this one get for $\Delta=\gamma^{i k} R,{ }_{i} R,{ }_{k}$ :

$$
\Delta=-\frac{A}{H^{2}} \quad \Longrightarrow \quad \frac{A}{H^{2}}=1-\frac{2 G m(z)}{r} .
$$

When $m=$ const, then the Vaidya metrics should be reduced to the Schwarzschild one, and from this it follows that that the metric coefficient $H$ should depend on the null coordinate $z$ only and by the transformation $z^{\prime}(z)$ can be made equal $\varepsilon$. Eventually, the Vaidya metrics takes the form

$$
d s^{2}=\left(1-\frac{2 G m(z)}{r}\right) d z^{2}+2 \varepsilon d z d r-r^{2}\left(d \vartheta^{2}+\sin ^{2} \vartheta d \varphi^{2}\right) .
$$

The only thing left is to evaluate the energy-momentum tensor of radiation, and for this we will use the Einstein equations in vectorial form. Rather simple but cumbersome calculations lead us to the following result: the only nonzero component is

$$
T_{z}^{r}=-\frac{1}{4 \pi r^{2}} \frac{d m}{d z}
$$


The trace of the energy-momentum tensor is zero, as it should be expected in the case of radiation. One obtains also that $H=H(z)$, and this shows that our lines of reasoning are not controversial.

Finally, we would like to say several words about the apparent horizons defined by

$$
\Delta=0 \quad \Longrightarrow \quad r_{H}=2 G m(z) .
$$

Is it null (as for the Schwarzschild metrics), time-like or space-like? To answer this question, let us calculate the square (Lorentzian) of the normal vector to the surfaces $\Delta=$ const:

$$
D=\gamma^{i k} \Delta,{ }_{i} \Delta,{ }_{k}=-\left(1-\frac{2 G m(z)}{r}\right)(\Delta, r)^{2}+2 \varepsilon \Delta,{ }_{r} \Delta_{z} .
$$

When $\Delta=0$, then

$$
D=-\varepsilon \frac{8 G^{2} m(z) m^{\prime}(z)}{r^{3}}
$$

Let us demand the energy dominance condition to be fulfilled. Then in the $R_{+}-$ region

$$
\operatorname{sign} m^{\prime}=-\varepsilon \quad \Longrightarrow \quad D>0 \text {. }
$$

Thus, the apparent horizon is space-like. In the $R_{-}$-region the $\varepsilon$ should be replaced by the $-\varepsilon$, so, the result remains the same. In particular, from this it follows that when the radiation is absorbing by a black hole, the apparent horizon as always lies beyond the event horizon, i.e., inside the resulting black hole. And this will be shown explicitly in the next, final Section.

\section{Static Observer-if it Exists in Reality, or only Virtually?}

Let us try to answer this question. What is the problem? The test particles we were used to think of as observers are virtual, having neither taste nor odor, i.e., with negligibly small masses and sizes. It may be thinkable (but not always) for the freely falling, along a geodesics, observer, but in the case of the static observer the situation is quite different. The point is that one should ensure such a statics in the gravitational field, and for this the external force is needed (what changes already the observed gravitational field) or to supply the observer with the rocket and fuel. But! First, it means that the additional mass appeared, not negligibly small, which also gravitates. Second, the fuel may be exhausted very soon (depending on its amount, i.e., on its mass), and then what?

To avoid the unnecessary complications with the gravitational radiation, we assume the spherical symmetry. Let us imagine the set (ensemble) of observers spread inside the narrow layer (in order to use the thin shell formalism) at some distance from an eternal black hole. To ensure the statics, we provide them with the photon rockets (the most effective ones) which will radiate the energy inside starting from some definite moment of time. Thus, outside we have the Schwarzschild metrics with the mass parameter $m_{\text {out }}=$ const, equal to the total mass (energy) of the system, and inside - the Vaidya metrics, written in advanced Finkelstein coordinates, with the varying mass $m_{\text {in }}(v)(\varepsilon=-1)$. The thin shell is at rest at the surface of constant radius $r=r_{0}$, i.e., in the Israel equations one should put $\rho=r_{0}, \dot{\rho}=\ddot{\rho}=0$. Again, for the sake of simplicity, we will consider a dust shell $\left(S_{2}^{2}=0\right)$, but now the bare mass is not constant more $-M=M(\tau)$. 
One more difference from the freely falling shell considered above: now the ingoing energy flow $T_{0}^{n}(\mathrm{in})$, and the normal component of the stress tensor $T_{n}^{n}(\mathrm{in})$, are not zero, their values are dictated by static condition.

And what do we have at hand? Israel equations

$$
\begin{gathered}
\sqrt{F_{\text {in }}}-\sqrt{F_{\text {out }}}=\frac{G M}{r_{0}}, \\
\frac{1}{F_{\text {in }}}\left(\frac{1-F_{\text {in }}}{2 r_{0}}+4 \pi G r_{0} T_{n}^{n}(\text { in })\right)-\frac{1-F_{\text {out }}}{2 r_{0} \sqrt{F_{\text {out }}}}=-\frac{G M}{r_{0}^{2}}, \\
\dot{M}=4 \pi r_{0}^{2} T_{0}^{n}(\text { in }), \\
F_{\text {in }}=1-\frac{2 G m_{\text {in }}(v)}{r_{0}}, \quad F_{\text {out }}=1-\frac{2 G m_{\text {out }}}{r_{0}}=F_{0}=\text { const },
\end{gathered}
$$

where we already took into account all our requirements (including $T_{0}^{n}$ (in) $=$ $\left.=T_{n}^{n}=0\right)$, and the energy-momentum tensor for the radiation

$$
T_{z z}=\varepsilon T_{z}^{r}=-\frac{\varepsilon}{4 \pi r_{0}^{2}} \frac{d m_{\mathrm{in}}}{d z},
$$

-all other components are zero. We need also to transform the Finkelstein coordinates $\{z, r\}$ to the Gaussian normal coordinates $\{\tau, n\}$, where the world line of the shell is just $n=$ const $=0$. Rather simple calculations lead us to the following result:

$$
\begin{gathered}
\frac{\partial z}{\partial \tau}=\frac{\sqrt{\dot{\rho}^{2}+F}-\varepsilon \dot{\rho}}{F}, \quad \frac{\partial z}{\partial n}=\frac{\dot{\rho}-\varepsilon \sqrt{\dot{\rho}^{2}+F}}{F}=-\varepsilon \frac{\partial z}{\partial \tau} \\
T_{n}^{n}=-\left(\frac{\sqrt{\dot{\rho}^{2}+F}-\varepsilon \dot{\rho}}{F}\right)^{2} T_{z z}, \quad T_{0}^{n}=-\varepsilon T_{n}^{n} .
\end{gathered}
$$

Since $\rho=r_{0}=$ const, we get

$$
T_{n}^{n}=\frac{\varepsilon}{4 \pi r_{0}^{2} F} \frac{d m}{d z}, \quad T_{0}^{n}=-\frac{1}{4 \pi r_{0}^{2} F} \frac{d m}{d z} .
$$

Now we have everything for answering the posed question. It follows from the continuity equation that

$$
\dot{M}=-\frac{1}{F_{\text {in }}} \frac{d m}{d v},
$$

and after some manipulations with the Israel equations,

$$
2 r_{0} \dot{M}=M\left(\frac{G M}{r_{0}}-A\right), \quad A=\frac{1-F_{0}}{\sqrt{F_{0}}} .
$$

This differential equation is easily solved:

$$
\frac{G M}{r_{0}}=\frac{A}{\exp \left(\frac{A\left(\tau-\tau_{0}\right)}{2 r_{0}}\right)+1},
$$

where $\tau_{0}$ is the integration constant, that is determined by the initial values of the bare mass, $M_{0}=M(\tau=0)$, initial mass inside the shell $m_{\text {in }}(\tau=0)$ and radius 
$r_{0}$ (the value of $F_{0}$ and, consequently, of $A$, can be derived from the first of Israel equations).

All the details, if one wishes, can be found in [23]. The last note. Please, look attentively at the Fig. 13 below. Here black horizontal lines are ordinary Schwarzschild singularities at zero radii, blue lines with slopes $\pm 45^{\circ}$ are future and past null infinities. Solid red curves are future and past apparent horizons where the the introduced in the paper invariant function $\Delta=0$, they separate the $R$-regions with $\Delta<0$ from $T$-regions with $\Delta>0, T_{+}$being the region of inevitable expansion, and $T_{-}$- the region of inevitable contraction. Black dashed curves represent hyper-surfaces of constant radii, they are time-like in $R$-regions and space-like in $T$-regions. The green solid curve shows a trajectory of our observers who first are freely falling and then, after switching on the engines of photon rockets (their ingoing radiation is indicated schematically by waving lines with arrows), keep themselves at rest at constant radius. The important feature of such a space-time is the future event horizon which is shown by the red dashed line with the slope $45^{\circ}$. Note, that the region confined within the event horizon and future and past apparent horizons is a part of the $R_{+}$-region where some curious person can stay infinitely long at constant radius or even go back to larger values of radii, but nevertheless he (she) is already inside the black hole and, thus unable to escape.

\section{REFERENCES}

1. J. Michell, "On the Means of Discovering the Distance, Magnitude, \&c. of the Fixed Stars, in Consequence of the Diminution of the Velocity of Their Light, in Case Such a Diminution Should be Found to Take Place in any of Them, and Such Other Data Should be Procured from Observations, as Would be Farther Necessary for That Purpose. By the Rev. John Michell, B. D. F. R. S. In a Letter to Henry Cavendish, Esq. F. R. S. and A. S." // Phil. Trans. R. Soc., 1784. Vol. 74. Pp. 35-57.

2. P.-S. Laplace, Le Système du Monde. Vol. II. Paris, 1799.

3. A. Einstein, "Zur Elektrodynamik bewegter Körper"// Ann. der Phys. (4), 1905. Vol. 17. 891-925 pp.

4. A. Einstein, "Über einen die Erzeugung und Verwandlung des Lichtes betreffenden heuristischen Gesichtspunkt" // Ann. der Phys. (4), 1905. Vol. 17. Pp. 132-148.

5. A. Einstein, "Zur Theorie der Lichterzeugung und Lichtabsorption" // Ann. der Phys. (4), 1906. Vol. 20. Pp. 199-206.

6. A. Einstein, "Ist die Trägheit eines Körpers von seinem Energieinhalt abhängig?" // Ann. der Phys. (4), 1905. Vol. 18. Pp. 639-641.

7. A. Einstein, "Über die vom Relativitätsprinzip geforderte Trägheit der Energie" // Ann. der Phys. (4), 1907. Vol. 23. Pp. 371-384.

8. K. Schwarzschild, "Über das Gravitationsfeld eines Massenpunktes nach der Einsteinschen Theorie" / In: Sitzungsberichte der Königlich Preussischen Akademie der Wissenschaften. Berlin: Königlich Preussische Akademie der Wissenschaften, 1916. Pp. 189-196; K. Schwarzschild, On the gravitational field of a mass point according to Einstein's theory, arXiv: physics/9905030 [physics.hist-ph].

9. L. D. Landau, E. M. Lifshitz, The Classical Theory of Fields / A Course of Theoretical Physics. Vol. 2. Oxford: Pergamon Press, 1971. xi+374 pp.

10. I. D. Novikov, " $R$ - and $T$-regions in space-time with a spherically symmetrical space" // Soobsh. GAISh, 1964. Vol. 132, no. 3. Pp. 3-42.

11. V. A. Berezin, V. A. Kuzmin, I. I. Tkachev, "Dynamics of bubbles in general relativity" // Phys. Rev. D, 1987. Vol. 36, no. 10. Pp. 2919-2944. 
12. V. A. Berezin, Vector-like Einstein's equations for D-dimensional spherical gravity with (D-2)-dimensional sphere, arXiv: gr-qc/0010083.

13. V. A. Berezin, A. L. Smirnov, "Crossing thin shells"// Grav. Cosmol., 2003. Vol.9, no. 4. Pp. 235-242.

14. D. Finkelstein, "Past-Future Asymmetry of the Gravitational Field of a Point Particle" // Phys. Rev., 1958. Vol. 110, no. 4. Pp. 965-967.

15. G. D. Birkhoff, Relativity and Modern Physics. Cambridge: Harvard University Press, 1923.

16. J. A. Wheeler, "Our Universe: The Known and the Unknown" // Amer. Sch., 1968. Vol. 37, no. 2. Pp. 248-274.

17. J. A. Wheeler, "Our Universe: The Known and the Unknown" // Am. Sci., 1968. Vol. 56, no. 1. Pp. 1-20.

18. J. A. Wheeler, "Our Universe: The Known and the Unknown" // Phys. Teach., 1968. Vol. 7, no. 1. Pp. 24-34.

19. A. Einstein, N. Rosen, "The Particle Problem in the General Theory of Relativity" // Phys. Rev., 1935. Vol. 48, no. 1. Pp. 73-77.

20. W. Israel, "Singular hypersurfaces and thin shells in general relativity" // Il Nuovo Cimento B Series 10, 1966. Vol.44, no. 1. Pp. 1-14.

21. W. Israel, "Singular hypersurfaces and thin shells in general relativity" // Il Nuovo Cimento B Series 10, 1967. Vol.48, no. 2. Pp. 463.

22. K. Kuchar̆, "Charged shells in general relativity and their gravitational collapse" // Czeck. J. Phys. B, 1968. Vol. 18, no. 4. Pp. 435-463.

23. V. Berezin, "Could a real (not virtual) static observer exist outside a Schwarzschild black hole?" // Gen. Rel. Grav., 2012. Vol.44, no. 6. Pp. 1555-1561, arXiv: 1106.0670 [gr-qc].

Original article submitted 10/VIII/2013;

revision submitted $22 / \mathrm{VIII} / 2013$. 